\title{
The dancing sky: 6 years of night-sky observations at Cerro Paranal ${ }^{\star}$
}

\author{
F. Patat \\ European Southern Observatory (ESO), K. Schwarzschildstr. 2, 85748, Garching b. München, Germany \\ e-mail: fpatat@eso.org
}

Received 19 December 2007 / Accepted 16 January 2008

\section{ABSTRACT}

\begin{abstract}
Aims. This work provides the results of the first six years of operation by the systematic night-sky monitoring at ESO-Paranal (Chile). Methods. The $U B V R I$ night-sky brightness was estimated on about 10000 VLT-FORS1 archival images, obtained on more than 650 separate nights, distributed over 6 years, and covering the descent from maximum to minimum of sunspot cycle n. 23. Additionally, a set of about 1000 low-resolution, optical, night-sky spectra were extracted and analysed.

Results. The unprecedented database discussed in this paper has led to detecting a clear seasonal variation of the broad-band nightsky brightness in the VRI passbands, similar to the well-known semi-annual oscillation of the Na I D doublet. The spectroscopic data demonstrate that this seasonality is common to all spectral features, with the remarkable exception of the $\mathrm{OH}$ rotational-vibrational bands. A clear dependency on the solar activity is detected in all passbands and is particularly pronounced in the $U$ band, where the sky brightness decreased by $\sim 0.6$ mag arcsec ${ }^{-2}$ from maximum to minimum of solar cycle $\mathrm{n}$. 23 . No correlation is found between solar activity and the intensity of the $\mathrm{Na}$ I D doublet and the $\mathrm{OH}$ bands. A strong correlation between the intensity of N I $5200 \AA$ and [OI]6300, $6364 \AA$ is reported here for the first time. The paper also addresses the determination of the correlation time-scales with solar activity and the possible connection with the flux of charged particles emitted by the Sun.
\end{abstract}

Key words. atmospheric effects - site testing - techniques: photometric - techniques: spectroscopic

\section{Introduction}

Soon after the beginning of VLT science operations in Paranal, ESO started an automatic UBVRI sky-brightness survey, with the aim of both characterising the site and studying the longterm trend, in order to detect any possible effects of human activity. The results obtained during the first 18 months of operations (April 2000-September 2001) were presented and discussed in Patat (2003a, hereafter Paper I). This program, which makes use of all scientific images obtained with FORS1, is building one of the most extensive, accurate, and homogeneous optical skybrightness data sets ever to be studied. As shown in Paper I, these data allow a very detailed analysis, including the study of correlations with other parameters, and the investigation of short, medium and long-term variations. The interested reader can find exhaustive reviews on this subject in Roach \& Gordon (1973) and in Leinert et al. (1998), while references to other published sky-brightness surveys are given in Paper I.

Since the publication of the first results, obtained on 174 different nights close to maximum of solar cycle n. 23, the data base has been steadily growing and progressively extending towards the solar minimum. In this paper, I present a global analysis run on the whole data set, which includes broad-band observations taken on 668 separate nights between 20 Apr. 2001 and 20 Jan. 2007. Additionally, I present and discuss here a set of more than 1000 low-resolution long-slit night sky spectra taken with FORS1 between May 1999 and Feb. 2005. They were used to measure the fluxes of single lines or integrated $\mathrm{OH}$ bands.

The paper is organised as follows. In Sect. 2 I describe the observations and the basic data reduction steps for photometry

^ Based on observations with ESO Telescopes at Paranal Observatory. and long slit spectroscopy. In Sect. 3 I review the general results obtained during dark time, while the correlation with solar activity and the seasonal variations are discussed in Sects. 4 and 5, respectively. The spectroscopic analysis is presented in Sects. 6-8. Finally, in Sect. 9 I discuss the main results and summarise the conclusions in Sect. 10.

\section{Observations and data reduction}

The data used in this work were obtained with the FOcal Reducer/low dispersion Spectrograph (hereafter FORS1), mounted at the Cassegrain focus of ESO-Antu/Melipal/Kueyen $8.2 \mathrm{~m}$ telescopes (Szeifert 2002). The instrument is equipped with a $2048 \times 2048$ pixel (px) TK2048EB4-1 backside, thinned CCD and has two remotely exchangeable collimators, which give a projected scale of $0^{\prime \prime} .2$ and $0^{\prime \prime} .1$ per pixel $(24 \mu \mathrm{m} \times 24 \mu \mathrm{m})$. According to the collimator used, the sky area covered by the detector is $6.8 \times 6 !^{\prime} 8$ and $3{ }^{\prime} 4 \times 33^{\prime} 4$, respectively.

\subsection{Photometry}

The photometric data set includes 10432 images obtained in the $U B V R I$ passbands with both collimators. The reduction procedure is described in Paper I, to which I refer the reader for a more detailed description, while here I only review the basic steps. All frames are automatically processed by the FORS pipeline, which applies bias and flat-field correction, the latter performed using twilight sky flats. Once the instrument signatures are removed from the images, the sky background is estimated using the robust algorithm described in Patat (2003b). The photometric calibration into the Johnson-Cousins system is then achieved 
Table 1. Main properties of the FORS1 spectral data set.

\begin{tabular}{cccccc}
\hline \hline Grism & Filter & $\begin{array}{c}\text { Wav. range } \\
(\AA)\end{array}$ & $\begin{array}{c}\text { Resolution } \\
(\AA F W H M)\end{array}$ & $\begin{array}{c}\text { Dispersion } \\
\left(\AA \mathrm{px}^{-1}\right)\end{array}$ & $N$ \\
\hline $300 \mathrm{~V}$ & $G G 435$ & $4300-8900$ & 12 & 2.6 & 676 \\
300V & - & $3615-8900\left(^{*}\right)$ & 12 & 2.6 & 163 \\
600B & $O G 590$ & $3650-6050$ & 5.3 & 1.2 & 143 \\
600R & $G G 435$ & $5390-7530$ & 4.5 & 1.0 & 207 \\
\hline
\end{tabular}

(*) Second-order overlapping above $6000 \AA$.

Note: only spectra taken with sun elevation below $-18^{\circ}$ were included.

using zeropoints and colour terms derived from the observation of standard star fields (Landolt 1992), regularly obtained as part of the FORS calibration plan. Finally, the observed values are corrected to zenith using the standard procedure (see for example Garstang 1989) and logged with a number of relevant parameters.

\subsection{Spectroscopy}

The spectroscopic data set includes a sub-sample of all longslit science data present in the ESO archive whose proprietary period had expired by the time this paper was written. For the sake of simplicity, I have selected only the data obtained with the standard resolution collimator and the single-port, high-gain read-out mode, since this combination is used the most for longslit spectroscopy with FORS1. To accumulate a wide data sample, I retrieved from the VLT archive all public spectra taken with the $300 \mathrm{~V}$ grism coupled with the order-sorting filter GG435 and a long slit $1^{\prime \prime} .0$ wide, which is the one most frequently used (676 frames). The wavelength range was extended down to about $3600 \AA$ in the blue by also retrieving all spectra taken with the same setup but with no order-sorting filter, for a total of 163 frames. To increase the sample, I retrieved also all spectra obtained with the grisms 600B (143 frames) and 600R (207 frames) coupled with the order-sorting filters OG590 and GG435, respectively. Also in these two cases the slit was 1".0 wide. The main characteristics of each setup are summarised in Table 1. Exposure times range from a few minutes to one hour.

All images were processed within the longslit package of IRAF $^{1}$. Due to the large amount of data and the purpose of this work, the bias subtraction was performed using only a pre-scan correction, while flat-fielding effects were neglected.

Wavelength calibration was achieved using a 2D solution derived from a set of reference arc exposures. Given the procedure adopted for the spectrum extraction, this step is mandatory, since in FORS1 the line curvature can reach a peak-to-peak value of about $10 \mathrm{px}$. If not accounted for, this instrumental feature would produce an apparently significant but artificial line broadening when collapsing the 2D spectra perpendicularly to the dispersion direction (see next section).

\subsubsection{Nightglow spectrum extraction and calibration}

After applying the appropriate 2D wavelength solution to all frames, the night-sky spectrum was extracted. For this purpose I used a robust algorithm to estimate the mode intensity in each column perpendicular to the dispersion direction. This implicitly

\footnotetext{
1 IRAF is distributed by the National Optical Astronomy Observatories, which are operated by the Association of Universities for Research in Astronomy, under contract with the National Science Foundation.
}

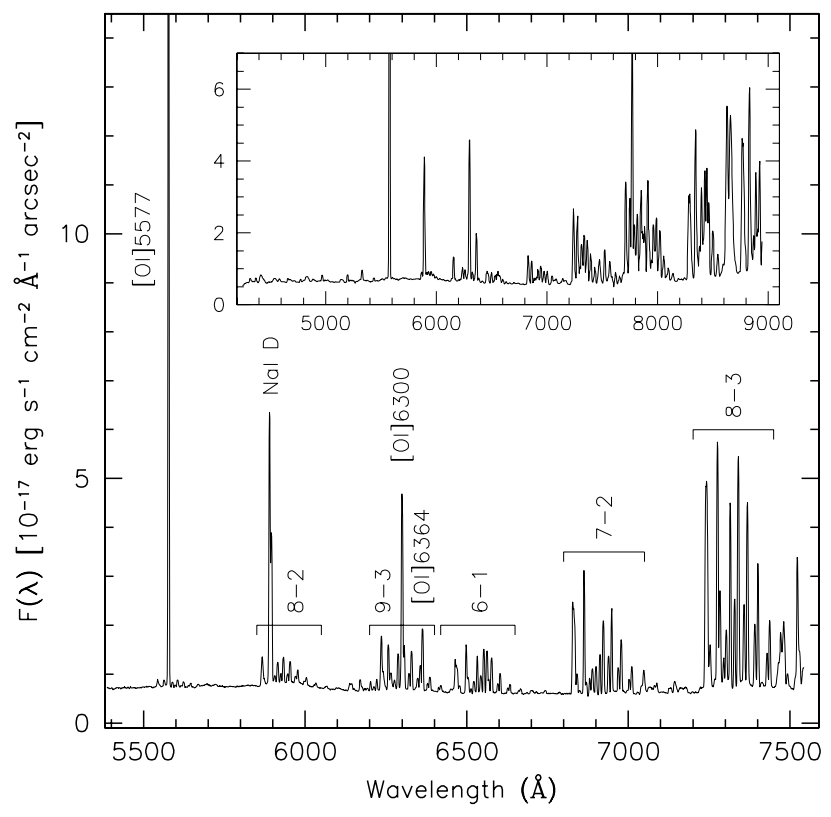

Fig. 1. Example of a FORS1 flux-calibrated night-sky spectrum obtained with the procedure outlined in the text, for the 600R grism. Main lines and $\mathrm{OH}$ bands identifications are given. The insert shows an example spectrum obtained with the $300 \mathrm{~V}$ grism.

assumes that most of the pixels are not "contaminated" by the contribution of astrophysical objects, which is reasonable in the majority of the cases, as verified by direct inspection of the whole two-dimensional data sample. This is both a consequence of the relatively large slit length featured by FORS1 (6.'8 on 2048 px) and the typical targets observed with this instrument, which are very often faint and star-like sources. After visual inspection, only a few spectra were removed from the final data set.

To allow for completely unsupervised line and continuum flux measurements, the accuracy of wavelength calibration is a mandatory requirement. Possible causes of rigid shifts in the dispersion solution can be identified as instrument interventions, turning into movements of the long slit on the focal plane, and flexures at large zenith distances. To correct for these problems, I produced a reference night sky spectrum for each of the two resolutions I have used, with a typical accuracy (estimated on isolated lines) better than $1 \AA$. Then, by means of cross-correlation, the zero point of the wavelength scale of each spectrum is automatically corrected at the end of the extraction procedure. This ensures that, at this stage, all spectra have maximum wavelength errors that do not exceed $1 \AA$.

For the absolute flux calibration I used a set of spectrophotometric standard stars to derive a reference sensitivity function $s(\lambda)$, which I applied to all spectra. Even though this does not take into account the changes in sensitivity which are mainly due to the ageing of reflective surfaces (Patat 2003a), at the wavelengths of interest they are a few percent, and therefore can be safely neglected in this context.

The flux calibration of the extracted spectrum $f(\lambda)$ to physical units is finally computed as

$$
F(\lambda)=\frac{f(\lambda)}{s(\lambda) t w p} \operatorname{erg~s}^{-1} \mathrm{~cm}^{-2} \AA^{-1} \operatorname{arcsec}^{-2}
$$

where $t$ is the exposure time (in seconds), $w$ the slit width (in $\operatorname{arcsec})$ and $p$ the projected pixel scale $\left(\operatorname{arcsec} \mathrm{px}^{-1}\right)$. 


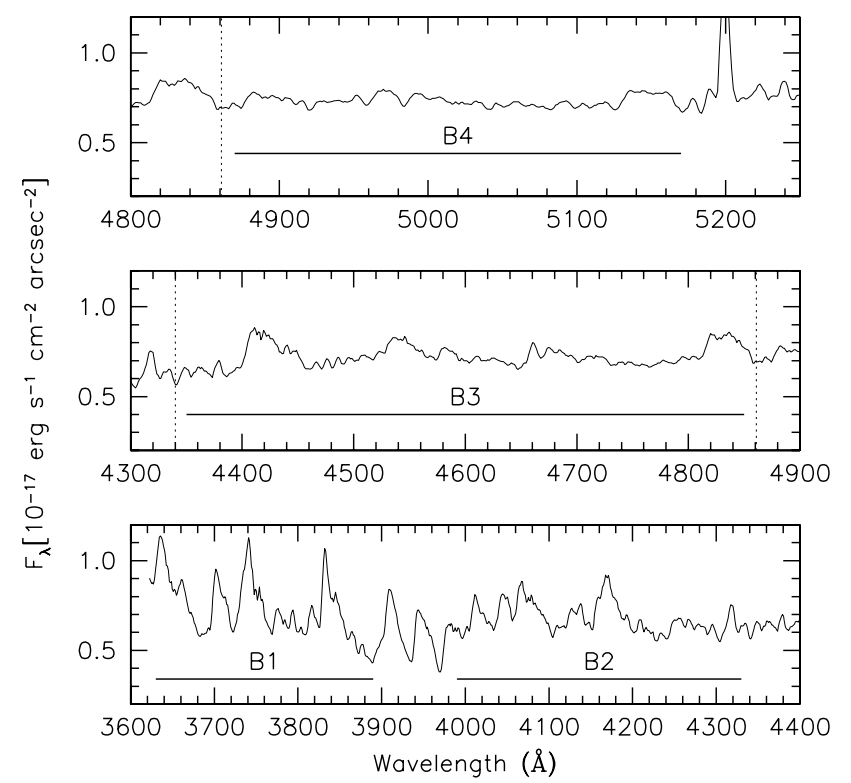

Fig. 2. Definition of the blue bands B1, B2, B3, and B4.

An example of flux-calibrated spectra obtained with this procedure is presented in Fig. 1. The resulting signal-to-noise ratio on the pseudo-continuum changes according to the exposure time of the original frame, but it is always higher than 100 .

\subsubsection{Line/bands flux measurements}

The line-flux integration is carried out in a fully automated way within a given wavelength window after subtracting the estimated pseudo-continuum intensity. The integration boundaries have been set according to the spectral resolution. For single lines, like the $[\mathrm{OI}] 5577$, the optimal window semi-amplitude has been set to $1.7 \times F W H M$, which corresponds to a $\pm 4 \sigma$ from the line centre. The pseudo-continuum level is estimated in the adjacent emission line-free regions. For more complex features (Na ID doublet, $\mathrm{OH}$ bands), the integration boundaries and the continuum region/s have been set ad hoc and are reported in Table 2.

Following Barbier (1956), I also introduced four bands, indicated as B1, B2, B3, and B4, in the blue spectral domain (see Table 2). The gap between bands B1 and B2 was set to avoid the strong Ca II H\&K absorptions (Fig. 2). Finally, a broad-band ranging from $5500 \AA$ to $7530 \AA$ was introduced, with the main aim of giving an overall flux estimate.

As in the case of the broad-band measurements, the line fluxes need to be corrected for airmass. Between the two cases, however, there is a difference: in fact, while the integrated flux within a broad-band filter is the result of extra-terrestrial sources (zodiacal light, unresolved stars, and galaxies) and emission within the atmosphere, all the radiation is of terrestrial origin in the case of nightglow emission lines. Practically speaking, this coincides with setting $f=1$ in Eq. (C.3) of Paper I, which I used to correct the observed values.

In general, the signal in the measured features is so high that the uncertainty in the line fluxes is by far dominated by the contamination by unresolved $\mathrm{OH}$ lines and, to a smaller extent, to the uncertainty on the pseudo-continuum level.
Table 2. Integration boundaries and continuum regions used for line flux measurements.

\begin{tabular}{ccccc}
\hline \hline Line/Band & Line range & Cont. range & Setup \\
\hline NI 5200 & $\pm 4 \sigma$ & $5040-5120$ & $(\mathrm{C} 1)$ & $300 \mathrm{~V}, 600 \mathrm{~B}$ \\
OI] 5577 & $\pm 4 \sigma$ & $5480-5520$ & $(\mathrm{C} 2)$ & $300 \mathrm{~V}, 600 \mathrm{~B} / \mathrm{R}$ \\
Na I D & $\mathrm{D}_{2}-4 \sigma-\mathrm{D}_{1}+4 \sigma$ & $5800-5850$ & $(\mathrm{C} 3)$ & $300 \mathrm{~V}, 600 \mathrm{~B} / \mathrm{R}$ \\
$\mathrm{OI}$ 6300 & $\pm 4 \sigma$ & $6400-6450$ & $(\mathrm{C} 4)$ & $300 \mathrm{~V}, 600 \mathrm{R}$ \\
$\mathrm{OI}$ 6364 & $\pm 4 \sigma$ & $6400-6450$ & & $300 \mathrm{~V}, 600 \mathrm{R}$ \\
\hline $\mathrm{OH}(6-1)$ & $6435-6680$ & $6750-6800$ & $(\mathrm{C} 5)$ & $300 \mathrm{~V}, 600 \mathrm{R}$ \\
$\mathrm{OH}(7-2)$ & $6810-7060$ & $6750-6800$ & & $300 \mathrm{~V}, 600 \mathrm{R}$ \\
$\mathrm{OH}(8-3)$ & $7200-7450$ & $6750-6800$ & & $300 \mathrm{~V}, 600 \mathrm{R}$ \\
$\mathrm{OH}(6-2)$ & $8250-8570$ & $8160-8230$ & $(\mathrm{C} 6)$ & $300 \mathrm{~V}+\mathrm{GG}$ \\
$\mathrm{O}_{2}(0-1)$ & $8605-8695$ & $8160-8230$ & & $300 \mathrm{~V}+\mathrm{GG}$ \\
\hline B1 & $3630-3890$ & - & & $300 \mathrm{~V}, 600 \mathrm{~B}$ \\
$\mathrm{~B} 2$ & $3990-4330$ & - & & $300 \mathrm{~V}, 600 \mathrm{~B}$ \\
$\mathrm{~B} 3$ & $4350-4850$ & - & $300 \mathrm{~V}, 600 \mathrm{~B}$ \\
B4 & $4870-5170$ & - & & $300 \mathrm{~V}, 600 \mathrm{~B}$ \\
\hline Broad-band & $5500-7530$ & - & $300 \mathrm{~V}, 600 \mathrm{R}$ \\
\hline
\end{tabular}

Table 3. Zenith-corrected average sky brightness during dark time at Paranal.

\begin{tabular}{cccccccc}
\hline \hline Filter & Sky Br. & $\sigma$ & Min & Max & $N_{\text {dark }}$ & $\Delta m_{\mathrm{ZL}}$ & $N_{\text {tot }}$ \\
\hline$U$ & 22.35 & 0.19 & 21.89 & 22.78 & 129 & 0.20 & 264 \\
$B$ & 22.67 & 0.16 & 22.19 & 23.02 & 493 & 0.28 & 1400 \\
$V$ & 21.71 & 0.24 & 21.02 & 22.30 & 692 & 0.20 & 1836 \\
$R$ & 20.93 & 0.24 & 20.42 & 21.56 & 1285 & 0.16 & 3931 \\
$I$ & 19.65 & 0.28 & 18.85 & 20.56 & 1137 & 0.07 & 3001 \\
\hline Total & & & & & 3736 & & 10432 \\
\hline
\end{tabular}

Note: Values are expressed in mag $\operatorname{arcsec}^{-2}$. Columns 3 to 8 show the rms deviation, minimum and maximum brightness, number of darktime data points, expected average contribution from the zodiacal light, and total number of data points.

\section{Dark-time night-sky brightness}

Since the data set includes observations obtained under a wide variety of conditions, it is necessary to apply some filtering in order to estimate the zenith sky brightness during dark time. To this aim I adopted the same criteria described in Paper I: photometric conditions, airmass $X \leq 1.4$, galactic latitude $|b|>10^{\circ}$, helio-eclitpic longitude $\left|\lambda-\lambda_{\odot}\right| \geq 90^{\circ}$, time distance from the closest twilight $\Delta t_{\mathrm{twi}}>1$ hour, and no moon (fractional lunar illumination FLI $=0$ or moon elevation $h_{\mathrm{M}} \leq-18^{\circ}$ ). The results of this selection, which reduced the number of suitable data points to 3736 , are summarised in Table 3 . As one can see, the average values are all within 0.1 mag of those reported in Paper I (see Table 4). In all filters there is a systematic shift towards darker values, with the only exception being the $I$ band. Since the values reported in Paper I were obtained during the sunspot maximum and, given the correlation between solar activity and night-sky brightness shown by Walker (1988), Pilachowski et al. (1989), Krisciunas (1990), Leinert et al. (1995), Mattila et al. (1996), Krisciunas (1997), and Krisciunas et al. (2007), this behaviour was indeed expected. Given the time distribution of data points (see Fig. 3), the average values reported in Table 3 are biased towards the sunspot maximum phase. For solar activity corrected data, see the next section.

Single measurements for the 5 passbands are presented in Fig. 4, which also show the surface-brightness distributions for the dark time and global samples. 


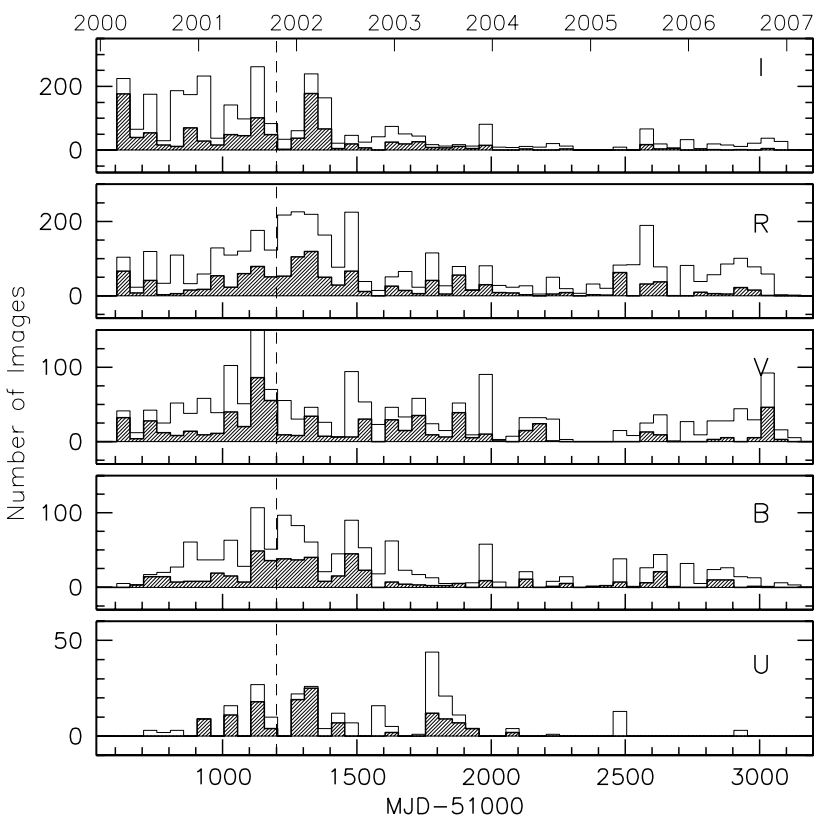

Fig. 3. Image distribution along the time interval covered by the present work for the global data set (thin line) and for the dark-time set (shaded thick line). The vertical dotted line marks the extent of the sample presented in Paper I, while the upper scale marks the January 1 of each year.

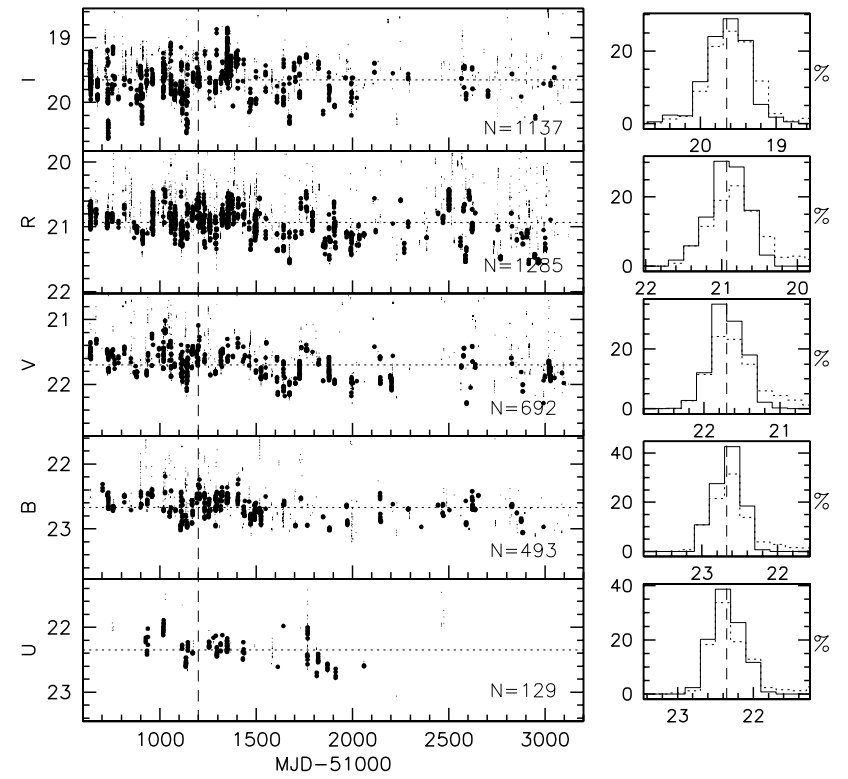

Fig. 4. Zenith-corrected sky brightness measured at Paranal during dark time (thick dots) from April 1, 2000 to April 8, 2006. The selection criteria are: $|b|>10^{\circ},\left|\lambda-\lambda_{\odot}\right| \geq 90^{\circ}, \Delta t_{\text {twi }}>1 \mathrm{~h}, \mathrm{FLI}=0$, or $h_{\mathrm{m}} \leq-18^{\circ}$. Thin dots indicate all observations (corrected to zenith). The horizontal dotted lines are positioned at the average values of the selected points, while the vertical dashed line marks the end of the time interval discussed in Paper I. The histograms trace the distribution of selected measurements (solid line) and all measurements (dotted line), while the vertical dashed lines are placed at the average sky brightness during dark time.

\section{Sky brightness vs. solar activity}

As first pointed out by Rayleigh (1928) and confirmed later by several other authors (see for instance Rosenberg \& Zimmerman 1967; Walker 1988; Krisciunas 1990; Leinert et al. 1995; Mattila et al. 1996; Krisciunas 1997; Krisciunas et al. 2007), many of

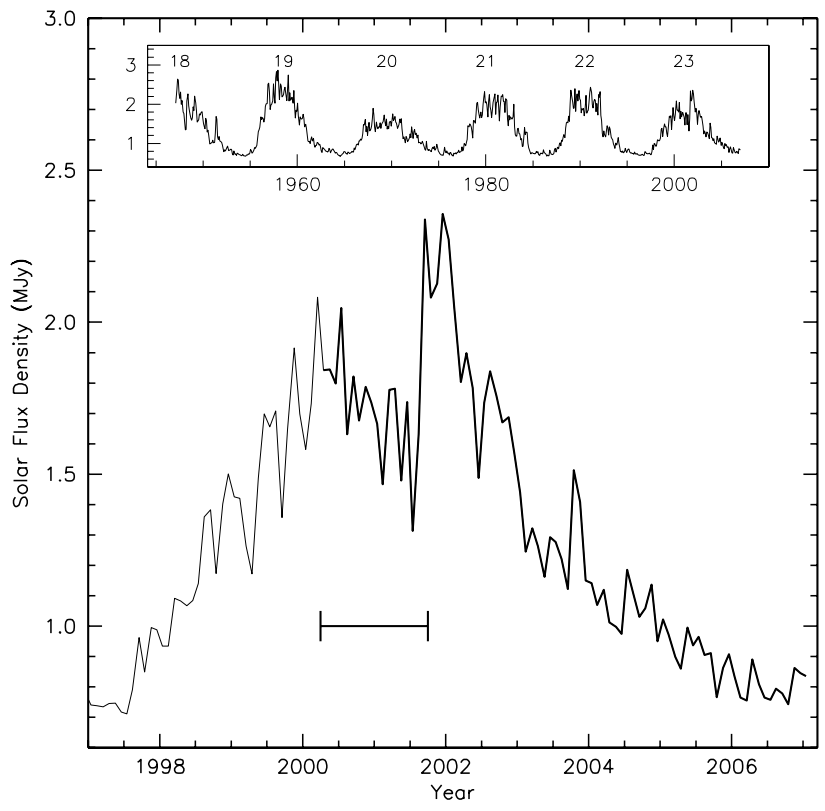

Fig. 5. Penticton-Ottawa Solar flux at $2800 \mathrm{MHz}$ (monthly average). The time range covered by the data presented in this paper is indicated by the thick line, while the horizontal segment indicates the time covered by the data presented in Paper I. The upper insert traces the solar flux during the last six cycles.
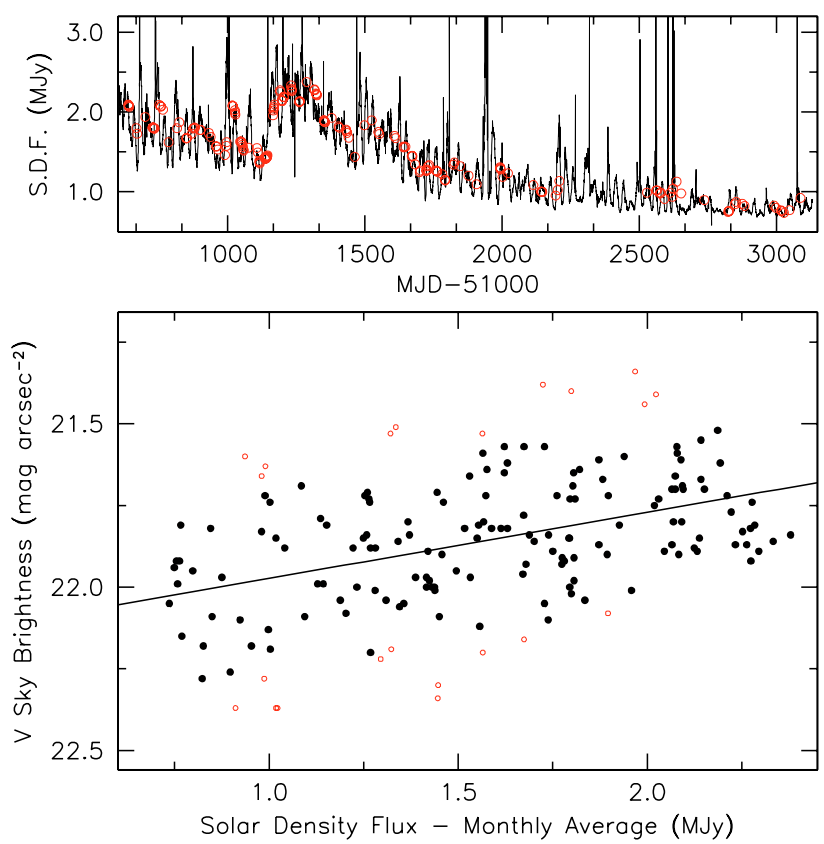

Fig. 6. Lower panel: nightly average dark-time sky brightness in the $V$ passband vs. solar flux density. The solid line is a linear least-square fit to the data (empty symbols mark the data points rejected by a kappasigma clipping). Upper panel: Penticton-Ottawa solar flux at $2800 \mathrm{MHz}$ during the time interval discussed in this paper. The open circles indicate the monthly averaged values corresponding to the $V$ nightly averages plotted in the lower panel.

the emission features in the night-sky spectrum show a clear dependency on the sunspot cycle. In particular, $B$ and $V$ present a peak-to-peak variation of $\sim 0.5 \mathrm{mag} \operatorname{arcsec}^{-2}$ during a full solar cycle. Less clear is the behaviour at longer wavelengths, which are dominated by the $\mathrm{OH}$ emissions, whose intensity is uncorrelated with solar activity (see Sect. 6). 
The data presented here cover the descent from the maximum of sunspot cycle n. 23 to the minimum phase, as shown in Fig. 5, which displays the monthly averaged Penticton-Ottawa solar flux at $2800 \mathrm{MHz}$ (Covington 1969) $)^{2}$. During this interval the solar flux density (hereafter $S F D$ ) spans from 0.8 to $2.4 \mathrm{MJy}$, a range which is very close to full cycle (the solar minimum is expected for the end of 2007). Following what has been done by other authors (see for instance Leinert et al. 1995), I studied the correlation between the sky brightness nightly averages and the SFD monthly averages, computed during the 30 preceding days. The sky-brightness measurements were corrected for the zodiacal light contribution computed for each data point as in Paper I (Sect. 4) and using the data by Levasseur-Regourd \& Dumont (1980).

All passbands show very good linear correlations, an example of which is presented in Fig. 6 for the $V$ filter. To give a quantitative representation of the effect, I fitted a relation of the type $m=m_{0}+\gamma S F D$ to the data. The results are shown in Table 4 for all filters. Besides reporting the zeropoint $\left(m_{0}, \mathrm{mag} \operatorname{arcsec}^{-2}\right)$, the slope $\left(\gamma\right.$, mag $\left.\operatorname{arcsec}^{-2} \mathrm{MJy}^{-1}\right)$, and their associated statistical errors, the table includes also the estimated full solar cycle variation $(\Delta m=|(2.4-0.8) \gamma|)$, the value attained at solar minimum, evaluated for $S F D=0.8 \mathrm{MJy}\left(m_{\min }\right)$, the value corresponding to the average $S F D$ level $\langle S F D\rangle=1.6 \mathrm{MJy}\left(m_{\text {ave }}\right)$, the rms deviation from the best fit relation $(\sigma)$, the linear correlation factor $(r)$, and the number of nights used $(N)$ for each filter.

As one can see, the values of $\Delta m$ are lower than those reported by other authors: with the only exception of $U$, which reaches about $0.6 \mathrm{mag} \operatorname{arcsec}^{-2}$, all the others show values that are lower than $0.4 \mathrm{mag} \operatorname{arcsec}^{-2}$. Walker (1988) quoted maximum ranges of $\Delta V \simeq 1.0$ and $\Delta B \simeq 0.8 \mathrm{mag} \operatorname{arcsec}^{-2}$ for solar cycle n. 21, while Krisciunas (1997) reports $\Delta V=0.6$ for solar cycle n. 22, and similar values are reported by Leinert et al. (1995) and Mattila et al. (1996). On the other hand, Liu et al. (2003) quote an increase in the $V$ sky brightness of $\sim 0.2 \mathrm{mag} \mathrm{arcsec}^{-2}$ from 1995 to 2001 . This value is consistent with the measures discussed here, especially taking into account that cycle n. 23 had a second maximum, which occurred after the observations presented by Liu et al. (2003). These facts seem to suggest that not all solar cycles have identical effects on the night glow. As a matter of fact, while revising the result of previous works, Walker (1988) suggested that the relation between intensity of the [OI] $5577 \AA$ line and the solar activity might vary from cycle to cycle, within a given cycle, and possibly with geographical location. Unfortunately, the number of sunspot cycles covered by the observations is still too small to allow a firm conclusion, but the very recent results discussed by Krisciunas et al. (2007), covering two full solar cycles, seem indeed to confirm this suspicion.

\subsection{Solar activity correlation time-scales}

Given the unprecedentedly large amount of data, one can investigate the solar dependency in a bit more detail, for example trying to deduce the typical time-scales of night-sky brightness fluctuations due to short-term changes in the solar flux. Ideally, to do this, one would look for strong enhancements in the solar flux and try to seek corresponding increases in the night-sky brightness. Nevertheless, due to the sparse time sampling, the only viable approach is actually the opposite one, i.e. starting from the available night-sky measurements, one goes back to the

2 The data are available in digital form at the following web site: http://www.drao.nrc.ca/icarus/www/archive.html
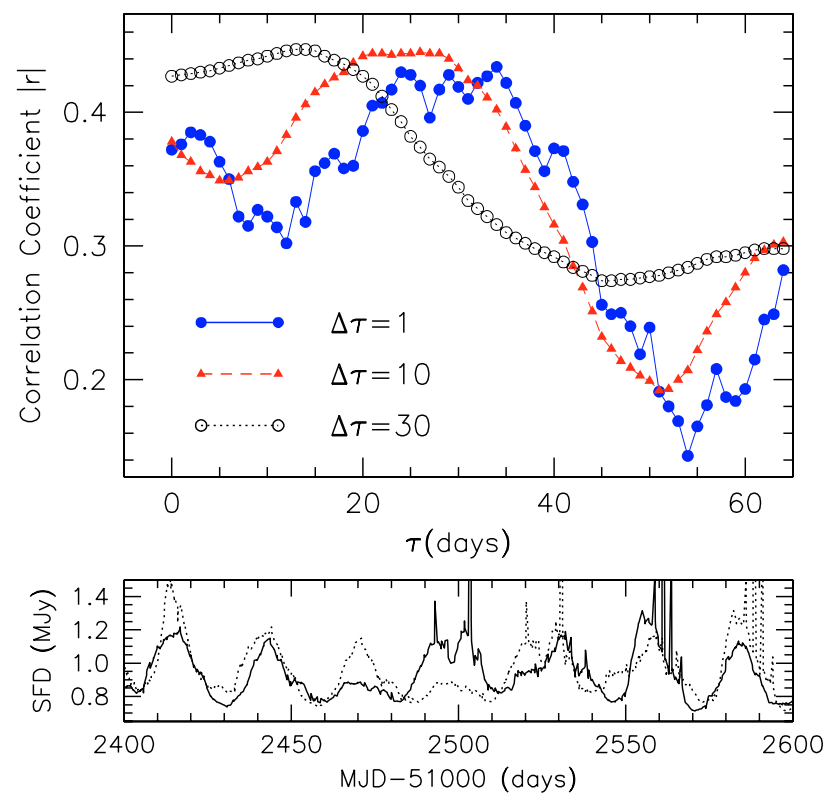

Fig. 7. Upper panel: linear correlation coefficient (absolute value) as a function of time delay $\tau$ for the $V$ passband data, computed for three different values of $\Delta \tau$ (1, 10, and 30 days). Lower panel: example of SFD periodicity due to solar rotation. The dotted curve is a replica of the original data shifted by 27.3 days.

solar data and studies the correlation with the sun flux emitted as a function of time lag. For doing this, I computed the Pearson's linear correlation coefficient $r$ (Press et al. 1992) between the nightly average sky brightness $m(t)$ measured at any given time $t$ and the solar flux density $\operatorname{SFD}(t-\tau)$, averaged within a time window $\Delta \tau$, as a function of both $\tau$ and $\Delta \tau$. While the first parameter indicates the time lag between a change in the solar flux and the consequent variation in the night-sky brightness, the second is related to the typical time-scales of the physical processes that govern the energy release in the upper atmosphere.

An example of this kind of analysis is shown in the upper panel of Fig. 7, which illustrates the behaviour of the correlation coefficient $r$ for the $V$ data, obtained on 170 separate nights for three different values of $\Delta \tau(1,10$, and 30 days). The correlation peak is quite broad and is centred at $\tau \sim 30$ days; moreover, the maximum correlation tends to increase slightly for larger averaging windows. This plot also explains why the average solar flux computed in the 30 days before the night-sky observations gives a better correlation than the sun flux measured on the preceding day, as already pointed out by several authors (see for example Leinert et al. 1995; Mattila et al. 1996). Finally, Fig. 7 clearly shows that the correlation drops significantly for $\tau>40$ days, a fact that is common to all UBVRI passbands.

An interesting feature to be noticed in Fig. 7 (seen also in the equivalent plots for the other filters) is the presence of spurious correlation peaks at a constant separation of about 27 days from the main peak. This is due to a periodicity in the solar flux data (see Fig. 7, lower panel), which is related to the solar rotation, whose synodic period is 27.3 days (Howard 1999). This semiregular recurrence in the solar data explains, for example, the two bumps close to $\tau=5$ and $\tau=65$ in Fig. 7 .

The strongest correlation is shown by the $U$ passband data, which presents a rather marked peak $r \sim 0.6$ at $\tau \sim 15$ days, while $r \sim 0.15$ for $\tau=1$ day. For this reason, averaging over the last 30 days gives a strong increase in the correlation, much stronger than in any other passband. A behaviour similar to what 
Table 4. Linear least-square fit parameters for the sky brightness vs. solar activity relation.

\begin{tabular}{ccccccccc}
\hline \hline Filter & $m_{\min }$ & $m_{\text {ave }}$ & $\Delta m$ & $m_{0}$ & $\gamma$ & $\sigma$ & $r$ & $N$ \\
\hline$U$ & 22.86 & 22.58 & 0.61 & $23.15(0.12)$ & $-0.36(0.07)$ & 0.15 & 0.47 & 32 \\
$B$ & 23.11 & 22.98 & 0.29 & $23.25(0.04)$ & $-0.17(0.02)$ & 0.12 & 0.40 & 127 \\
$V$ & 21.99 & 21.86 & 0.30 & $22.13(0.05)$ & $-0.17(0.03)$ & 0.14 & 0.42 & 148 \\
$R$ & 21.26 & 21.09 & 0.37 & $21.33(0.04)$ & $-0.22(0.02)$ & 0.15 & 0.44 & 202 \\
$I$ & 19.81 & 19.72 & 0.20 & $19.90(0.06)$ & $-0.11(0.04)$ & 0.18 & 0.28 & 144 \\
\hline
\end{tabular}

Note: Values in parenthesis indicate rms uncertainties. Input data have been corrected for the differential zodiacal light contribution (see text).

is displayed in $V$ is also seen in $B$ (correlation peak $r \sim 0.45$ for $\tau \sim 25$ days $)$ and $I(r \sim 0.30$ for $\tau \sim 20$ days $)$. Somewhat different is the case of the $R$ passband, for which the correlation peak $(r \sim 0.45)$ is attained at $\tau \sim 2$ days, suggesting that the sun-dependent features that contribute to the flux in this filter react rather rapidly to the solar flux fluctuations. In general, however, and with the possible exception of the $U$ passband, the correlation peaks are rather broad, indicating that different processes take place with different time-scales. The behaviour of the $U$ band, where the nightglow emission is dominated by the Herzberg and Chamberlain $\mathrm{O}_{2}$ bands (Broadfoot \& Kendall 1968), indicates that the photo-chemical reactions that are responsible for the emission in this region are more sensitive to solar activity.

\section{Seasonal variations}

In the previous work I had attempted to detect night sky brightness seasonal variations but, due to insufficient number of data points, I could not draw any firm conclusion (see Fig. 14 in Paper I). Thanks to the much larger sample available now, this analysis becomes feasible and, as a matter of fact, traces of a periodic modulation in the average sky brightness are visible already in Fig. 4. They become much clearer when each data point is plotted against the number of days from the beginning of the corresponding year. The result is shown in Fig. 8 where, besides reporting the single dark-time measurements, I have also plotted the monthly averages. The input data have been corrected for differential zodiacal light contribution and the solar flux dependency derived in the previous section has been removed using the parameters presented in Table 4.

This semi-annual oscillation (hereafter SAO) is definitely present in $V, R$, and $I$, while its presence in $B$ is more questionable ( $U$ data were not included since the sample in this passband is too poor for this purpose). The modulation amplitude grows at longer wavelengths, showing two maxima around April-May and October and two minima around July-August and December-January. In general, the variation is more pronounced in first half of the year than in the second one. For example, in $I$ it reaches a peak-to-peak value of about $0.5 \mathrm{mag} \operatorname{arcsec}^{-2}$.

As pointed out by Benn \& Ellison (1998), the variable contribution of zodiacal light can mimic a seasonal variation. To exclude a possible contribution by this source to the observed behaviour, I have analysed the expected enhancement of brightness due to the zodiacal light for each data point, using the data presented by Levasseur-Regourd \& Dumont (1980) and the procedure discussed in Paper I (Sect. 4) for the Paranal site. This does not show any significant regular pattern as a function of day of the year. The conclusion is that the observed SAO is not due to the periodic apparent variation in the ecliptic height above Paranal's horizon. Interesting is that the minima and maxima of

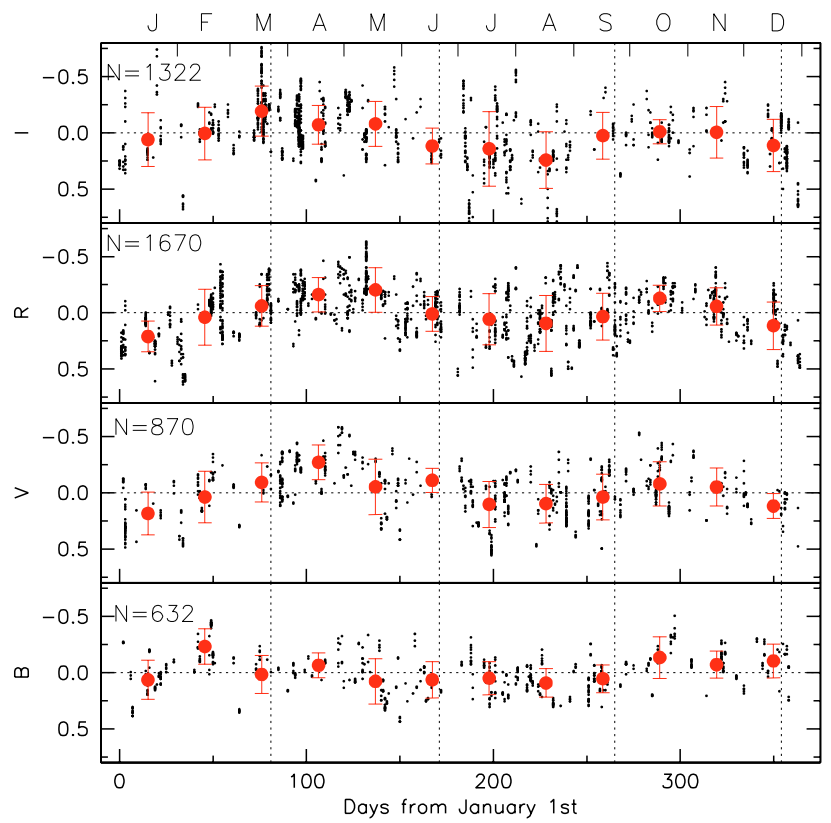

Fig. 8. Seasonal variation in dark-time sky brightness with respect to the average value. Small symbols are the single measurements, while the large symbols mark the monthly averages, computed in bins of 30 days each. The error bars indicate the rms deviations from the average within each bin, while the vertical dotted lines mark equinoxes and solstices. Data have been corrected for differential zodiacal light contribution and solar flux dependency.

the SAO occur out of phase with respect to the Equinoxes and Solstices (see Fig. 8, vertical dotted lines).

While seasonal variations of emission lines and/or bands have been studied by several authors in the past (see for example Chamberlain 1961; Roach \& Gordon 1973, and references therein), broad-band measurements are much more scanty and the results do not always agree. For instance, Schneeberger et al. (1979) report particularly bright values obtained in June at the Sacramento Peak Observatory and they find them to be "marginally correlated with the strong seasonal trend evident in the record of daytime sky brightness observations". In their survey run at the Lowell Observatory, Lockwood et al. (1990) discuss the seasonal variation, concluding that "neither winter enhancements [...] nor springtime rise [...] is indicated [...]". Benn $\&$ Ellison (1998) reach the same result from the analysis of the data obtained on La Palma, concluding that "dark-of-moon sky brightness does not vary significantly $(\leq 0.1 \mathrm{mag})$ with season [...]". Finally, when analyzing data taken at the Xinglong Station between 1995 and 2001, Liu et al. (2003) find that "the sky is darker in the fall and winter than in the spring and summer". While part of the discrepancies can be due to latitude effects (see for example Chamberlain 1961), some of the negative detections are probably to be ascribed to insufficient time sampling and coverage. In fact, the SAO amplitude is at most comparable with the night-to-night fluctuations and hence large and wellsampled data sets are required.

It is worth mentioning here that Garstang (1988) produced some simplified models to predict seasonal variations in the broad-band night-sky brightness, based on periodic variations in height and molecular density. The values predicted by these models are far too small with respect to those presented here, suggesting that other possible explanations must be investigated.

The observed behaviour might indicate that, whatever the reason for the periodic variation is, it is not directly related to 
Table 5. Observed line intensities and relative flux contribution to $V$ and $R$ passbands for [OI]5577, [OI]6300, and Na I D. All values are corrected to zenith.

\begin{tabular}{ccccc}
\hline \hline [OI]5577 & $\left(10^{-16} \mathrm{erg} \mathrm{s}^{-1} \mathrm{~cm}^{-2} \operatorname{arcsec}^{-2}\right)$ & $(R)$ & $V$ & $R$ \\
\hline $\min$ & 2.6 & 40 & $3 \%$ & $<1 \%$ \\
ave & 16 & 230 & $20 \%$ & $1 \%$ \\
$\max$ & 50 & 750 & $62 \%$ & $4 \%$ \\
\hline$[\mathrm{OI}] 6300$ & & & & \\
$\min$ & 0.6 & 10 & $<1 \%$ & $<1 \%$ \\
ave & 9 & 150 & $2 \%$ & $7 \%$ \\
$\max$ & 56 & 950 & $12 \%$ & $45 \%$ \\
\hline Na I & & & & \\
$\min$ & 0.7 & 10 & $<1 \%$ & $<1 \%$ \\
ave & 3 & 50 & $2 \%$ & $3 \%$ \\
$\max$ & 10 & 160 & $8 \%$ & $9 \%$ \\
\hline
\end{tabular}

Table 6. Linear least-square fit parameters for the line fluxes vs. solar activity relation.

\begin{tabular}{cccccc}
\hline \hline Line & $\log F_{0}$ & $\gamma$ & $\sigma$ & $r$ & $N$ \\
\hline OI 5577 & $-15.36(0.03)$ & $0.27(0.02)$ & 0.22 & 0.49 & 876 \\
OI 6300 & $-15.69(0.07)$ & $0.25(0.05)$ & 0.41 & 0.23 & 635 \\
Na I D & $-15.72(0.03)$ & $0.04(0.02)$ & 0.25 & 0.07 & 876 \\
NI 5200 & $-17.19(0.05)$ & $0.37(0.03)$ & 0.32 & 0.45 & 727 \\
\hline
\end{tabular}

Note: Values in parenthesis indicate rms uncertainties. Input data have been corrected to zenith.

As far as the solar activity is concerned, it must be noticed that even though a dependence on the $S F D$ is seen, it is less clear than in the case of the [OI]5577 (see Fig. 10, lower panel). In fact, even though the slopes are very similar, the linear correlation coefficient for [OI] 6300 is 0.23 , to be compared with 0.49 measured for the [OI]5577 line (see also Table 6).

It is well known that, during the first hours of the night, the [OI]6300 line shows a steady decline in brightness that, at tropical sites, is interrupted by abrupt emission bursts (see, for instance, Roach \& Gordon 1973). A plot of the line fluxes as a function of time elapsed after the evening twilight (see Fig. 12) clearly shows that these events occur during the whole night, and not only before midnight, as sometimes stated in the literature (see for instance Benn \& Ellison 1998). Actually, one interesting fact that emerges from this analysis is that, when the nights are short (filled symbols), i.e. during austral summer, most of the line enhancements tend to take place in the second half of the night. On the other hand, when the nights are long (i.e. lasting more than $8.5 \mathrm{~h}$ ), flux changes tend to become smaller and smaller during the last two hours of the night. No such dichotomy is observed for the [OI]5577 line (see Fig. 12, lower panel).

Finally, as for the broad-band data (see Sect. 4.1), I calculated the correlation coefficient between line fluxes and SFD for different values of the time delay $\tau$ and time window $\Delta \tau$. An instructive example is presented in Fig. 13, showing the case of the $[\mathrm{OI}] 5577$ line, which displays the strongest dependency on solar activity. The correlation function shows a peak at about $\tau=15$ days for $\Delta \tau=1$ day. The maximum correlation increases for $\Delta \tau=10$ days, peaking at $\tau \simeq 12.5$ days. Spurious correlation peaks due to the 27 days solar rotation are present as in the case of photometric data (see Fig. 7). A similar analysis for the $[\mathrm{OI}] 6300$ line shows two similar peaks at $\tau \simeq 13$ days and 27 days later.

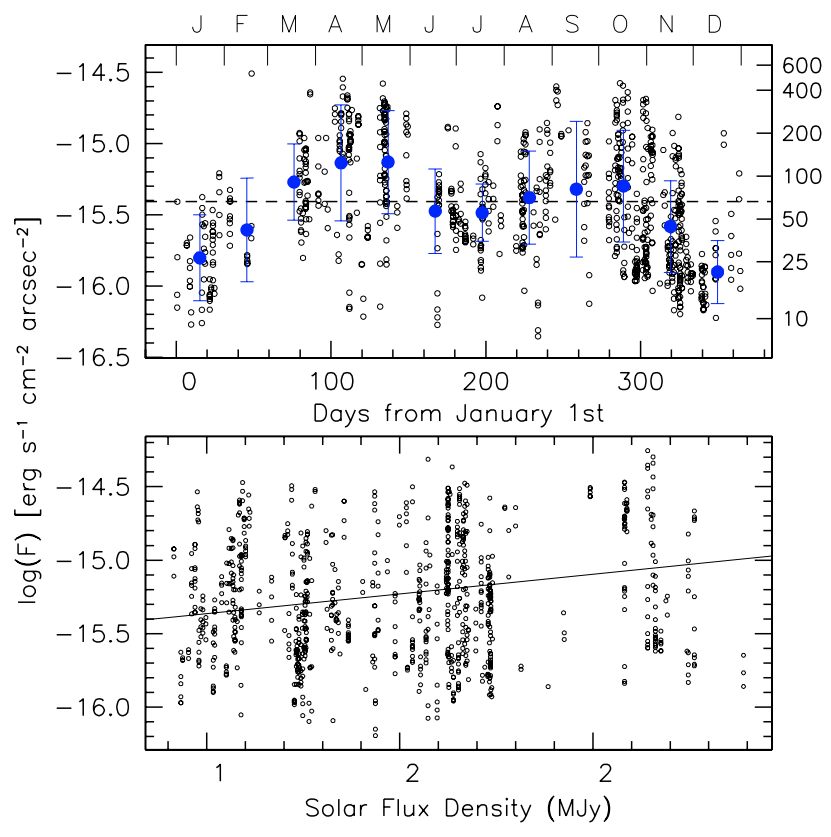

Fig. 10. Same as Fig. 9 for the $[\mathrm{OI}] 6300$ line.
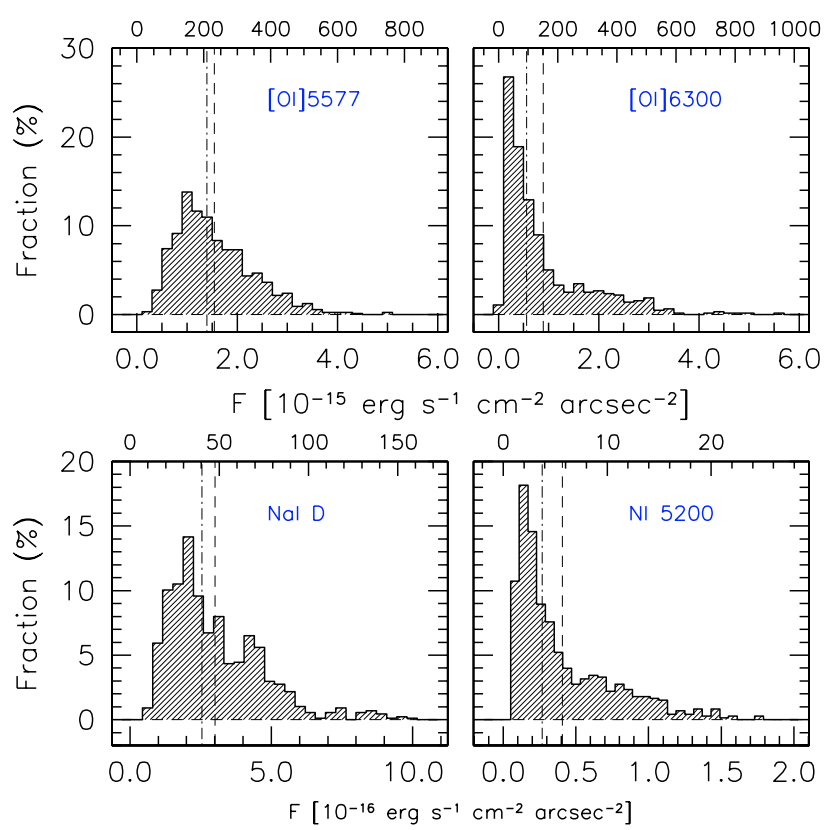

Fig. 11. Dark-time line flux distributions for [OI]5577 [OI]6300, $6364 \AA$, Na I D and N I 5200. The vertical lines trace the average (dashed) and the median (dotted-dashed) of the distributions. The upper horizontal scale in each panel is expressed in Rayleigh units.

\subsubsection{The $\mathrm{Na} I \mathrm{D}$ lines}

The Na I D doublet is known to originate in a layer placed at about $92 \mathrm{~km}$ and to undergo strong seasonal variations around an average value of 50 R (Roach \& Gordon 1973). For the Paranal site, the doublet varies by a factor greater than 15 , reaching a maximum value of $160 \mathrm{R}$ in April. The maximum contribution of the $\mathrm{Na}$ I $\mathrm{D}$ doublet to the global night sky brightness reaches $\sim 0.1$ mag $\operatorname{arcsec}^{-2}$ both in $V$ and $R$ passbands (see Table 5).

While the Na I D doublet intensity does not show any significant correlation with solar activity (the correlation coefficient is only 0.07; see Fig. 14 and Table 6), it does show a clear SAO, 


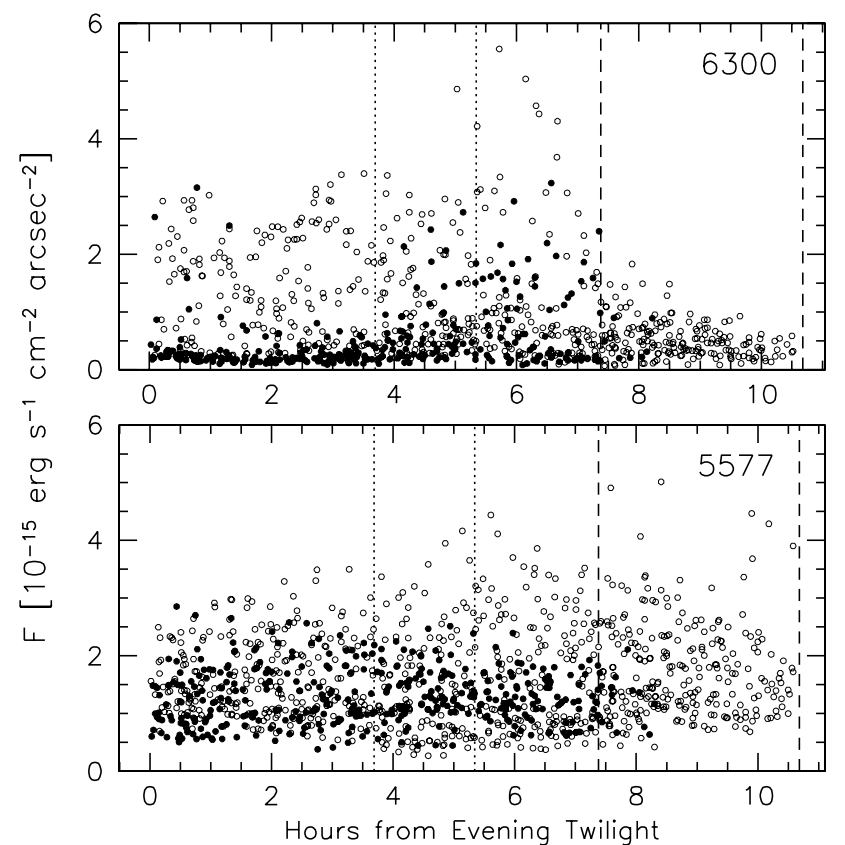

Fig. 12. Line fluxes as a function of time elapsed from evening twilight for [OI]6300 (upper panel) and [OI]5577 (lower panel). The vertical dashed lines mark the shortest and longest nights (7.4 and $10.7 \mathrm{~h}$, respectively), while dotted lines indicate the midnight in the two cases. Filled symbols indicate data obtained in nights whose length is between 7.4 and $8.4 \mathrm{~h}$.

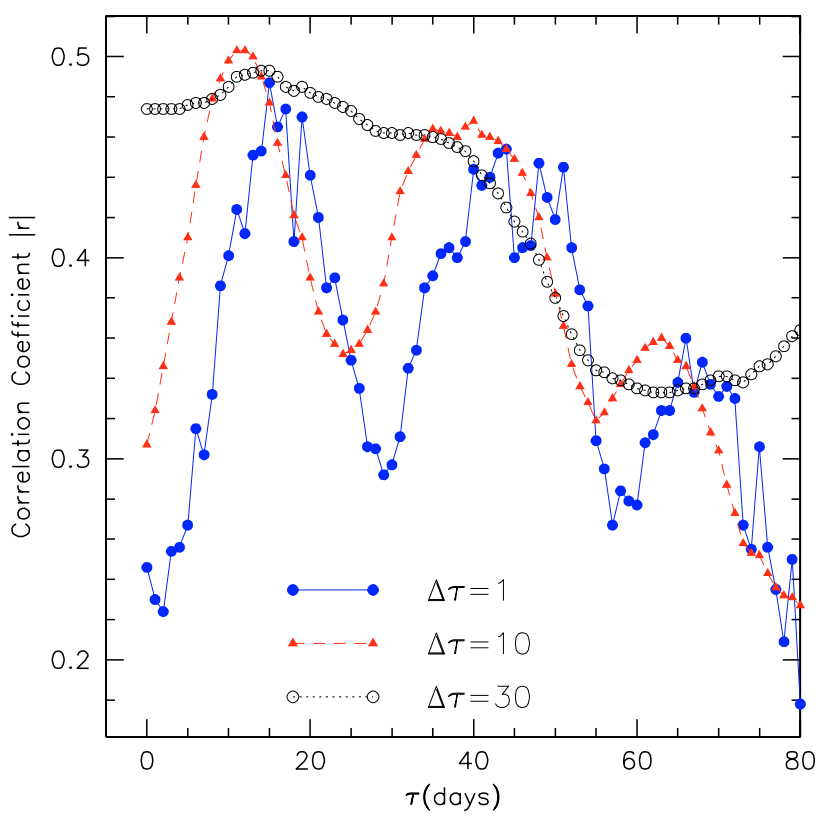

Fig. 13. Linear correlation coefficient (absolute value) as a function of time delay $\tau$ for the [OI]5577 line, computed for four different values of $\Delta \tau(1,10,30$ days $)$.

with a peak in April and a secondary peak in October/November (see Fig. 14, upper panel), as it is seen in all atomic lines analysed in this work. The SAO displayed by mesospheric sodium column density is a well-studied phenomenon (Kirchoff 1986), also because of the importance of the sodium layer for the laser guide star adaptive optics systems (see for instance Ageorges \& Hubin 2000). That different species like $\mathrm{Na}$ I and [OI] all show an SAO is interpreted in light of the role of $\mathrm{O}$ atoms in the source photochemical reactions (Slanger et al. 2005).

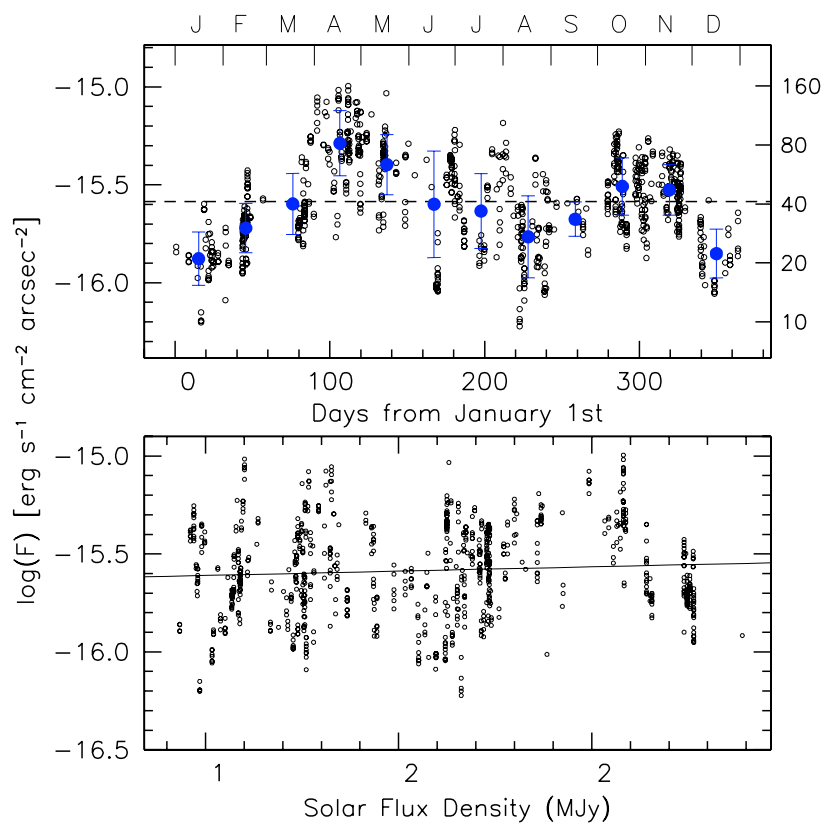

Fig. 14. Same as Fig. 9 for the Na I D doublet. In this case, no solar flux correction has been applied.

Since the resolution provided by the grism 600R is high enough to resolve the $\mathrm{D}_{1}$ and $\mathrm{D}_{2}$ components (see Fig. 15), following the work done by Slanger et al. (2005), I estimated the intensity ratio $D_{2} / D_{1}$ during dark time on a total of $147 \mathrm{spec}-$ tra. The region of interest is contaminated by the presence of two features belonging to the $\mathrm{OH}(8-2)$ band, which I indicate as $\mathrm{B}$ and $\mathrm{C}$ (see Fig. 15 for their identification). Using a highresolution UVES spectrum of the night sky (Hanuschik 2003), I estimated the intensity ratio between these two features and another $\mathrm{OH}(8-2)$ line at $5932.9 \AA\left(P_{11}(3.5)\right.$, Abrams et al. 1994), which I indicate as E. Since this feature is easily measured in the $600 \mathrm{R}$ spectra, assuming that these intensity ratios are constant $(B / E=0.95, C / B=0.35)$, I estimated the intensity of $B$ and $\mathrm{C}$ from the measured intensity of component $\mathrm{E}$. Then, after subtracting two Gaussian profiles centered at the laboratory wavelengths of $\mathrm{B}$ and $\mathrm{C}$ from the original data, I have fitted the residual with a double Gaussian profile, fixing the distance between the two components $(5.98 \AA)$ and their FWHM (4.5 $\AA$ ). Finally, I derived the $\mathrm{D}_{2} / \mathrm{D}_{1}$ by simply computing the ratio between the central intensities of the two fitted Gaussians. The pseudo-continuum was removed using a first order polynomial.

The results, as shown in Fig. 16 (upper panel), are perfectly in line with the findings published by Slanger et al. (2005): the intensity ratio, which should be 2.0 if the two transitions are produced according to their spin-orbit statistical weights, varies from 1.2 to 1.8 , with most of the data lying between 1.5 and 1.7. The average value for the FORS1 sample is 1.64 , with an rms deviation of 0.08 . Using a larger sample including more than 300 high-resolution spectra, Slanger et al. (2005) have found evidence of an SAO for the $\mathrm{D}_{2} / \mathrm{D}_{1}$ intensity ratio. This is not detected in the low-resolution data set presented here, but this is probably due to the present sample including less than half as many data points.

\subsubsection{The $\mathrm{N} I 5200$ line}

The N I feature at $\sim 5200 \AA$ is actually a blend of several transitions. It is supposed to originate at about $260 \mathrm{~km}$ altitude and has 


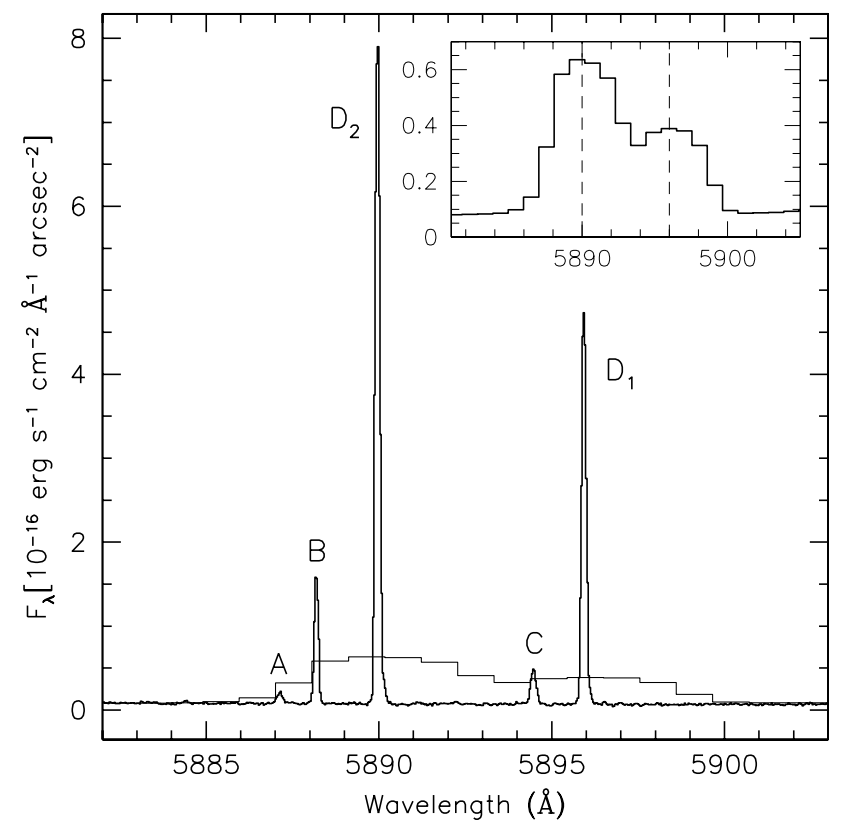

Fig. 15. Night-sky spectrum taken with UVES (Hanuschik 2003) in the region of the $\mathrm{Na}$ I D lines (resolution $0.15 \AA F W H M$. The labels A, $\mathrm{B}$, and C indicate the $\mathrm{OH}$ Meinel 8-2 band lines $Q_{22}(0.5) 5887.1 \AA$, $Q_{11}(1.5) 5888.2 \AA$ and $Q_{11}(2.5) 5894.5 \AA$ (Abrams et al. 1994). The insert shows the corresponding spectrum obtained with FORS 1 and the 600R grism (resolution $4.5 \AA F W H M$ ). The same spectrum is also shown in the main panel (thin line).
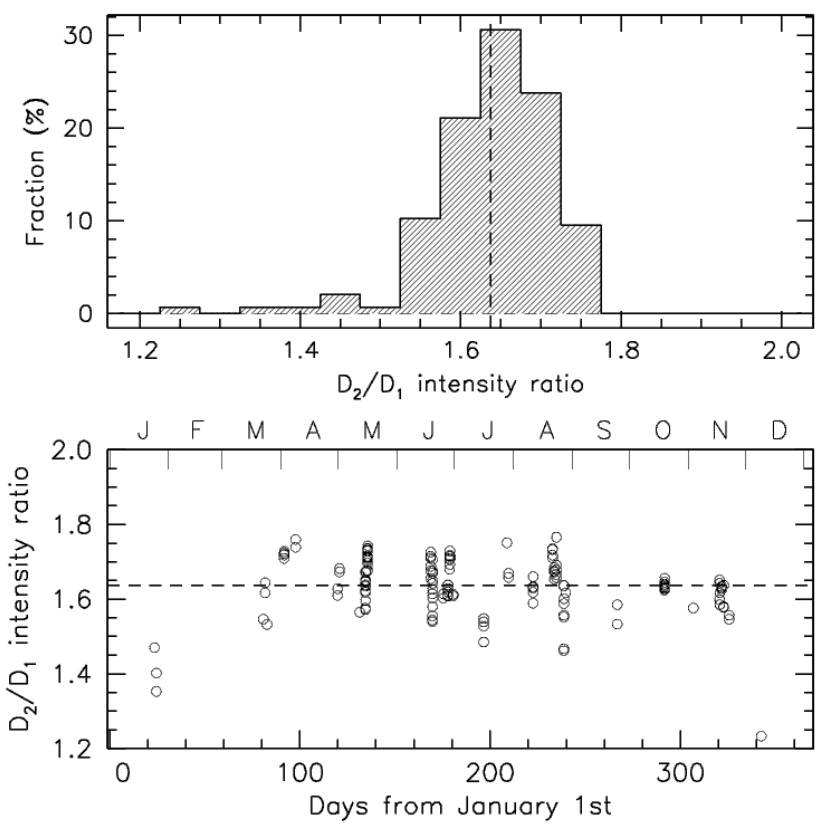

Fig. 16. Upper panel: distribution of the $\mathrm{Na} I \mathrm{D}_{2} / \mathrm{D}_{1}$ intensity ratio during dark time. Lower panel: $\mathrm{Na} I \mathrm{D}_{2} / \mathrm{D}_{1}$ intensity ratio as a function of time from the beginning of the year. The horizontal dashed line marks the average value.

a typical intensity of $1 \mathrm{R}$ (Roach \& Gordon 1973). As shown in Paper I, this line shows abrupt changes, possibly following the behaviour of the $[\mathrm{OI}] 6300$ line. The data discussed in this paper show that the flux of this line ranges from practically zero (the line is not detected) to about $30 \mathrm{R}$; moreover, the flux distribution is rather similar to that of [OI]6300 (see Fig. 11), strengthening the impression that these two lines are related. This line

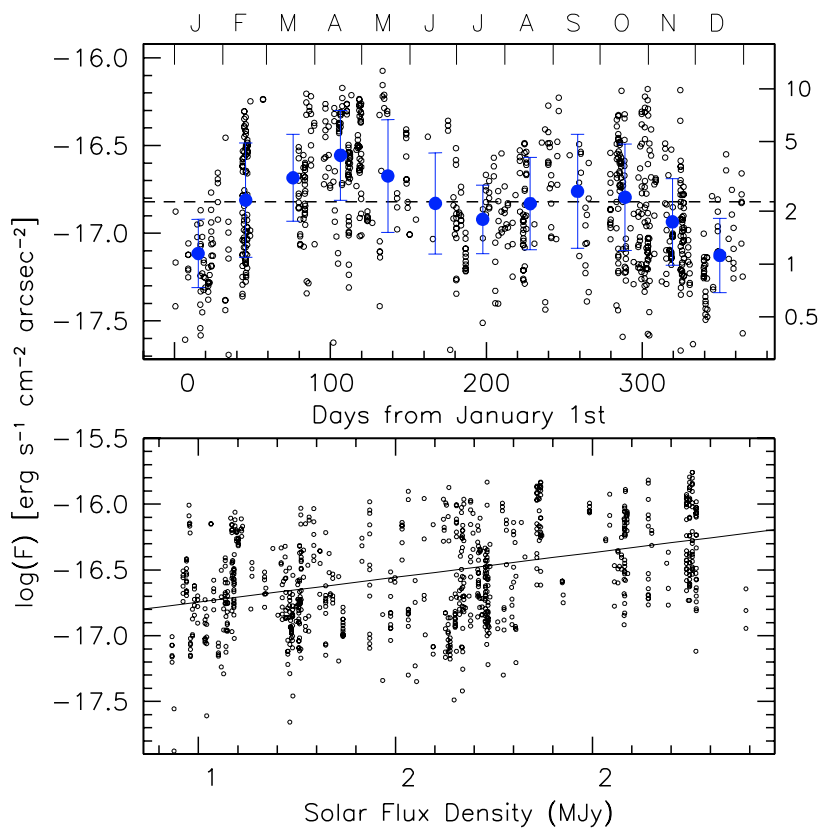

Fig. 17. Same as Fig. 9 for the N I 5200 A line.

also displays a strong dependency on solar activity, with a correlation factor similar to that of the [OI]5577 line (see Table 6). Finally, as for all other atomic lines discussed here, it shows an SAO (see Fig. 17). The maximum contribution of this line to the global flux in the $V$ band during dark time is about $2 \%$.

\subsection{Main molecular emission bands}

\subsubsection{The $\mathrm{OH}$ bands}

The $\mathrm{OH}$ bands analysed here (see Table 2) show a very tight mutual correlation, in that they appear to vary in unison. Moreover, they do not show any correlation with solar activity $(r \leq 0.1$ for all bands), while they show an SAO, even though not as pronounced as in the case of the other features discussed so far (see Fig. 18 for an example). The flux distribution appears to be much more symmetric around the average value than in the case of atomic lines. All bands shows the same range of variation, which is close to a factor 2 around the mean value. Given the intensity of these features (especially $\mathrm{OH}(8-3)$ and $\mathrm{OH}(6-$ $2)$ ), their variability is certainly the dominating source of sky brightness fluctuations in the I passband.

\subsubsection{The $\mathrm{O}_{2}(0-1)$ band}

This band shows a clear correlation with solar activity $(r=0.41)$ and the same SAO observed for all other features (see Fig. 19). Its integrated flux varies by about a factor 15 , between $80 \mathrm{R}$ and $1.1 \mathrm{kR}$.

\subsection{The blue bands and pseudo-continuum}

None of the blue bands, which are supposed to trace the behaviour of the pseudo-continuum generated by the $\mathrm{O}_{2}$ and $\mathrm{NO}_{2}$ molecular bands (Roach \& Gordon 1973), shows a clear dependency on solar activity $(r \leq 0.3)$. However, it must be noticed that, while the emission features are generated purely within the atmosphere, the continua are influenced significantly by the extra-terrestrial background (Roach \& Gordon 1973), which is 


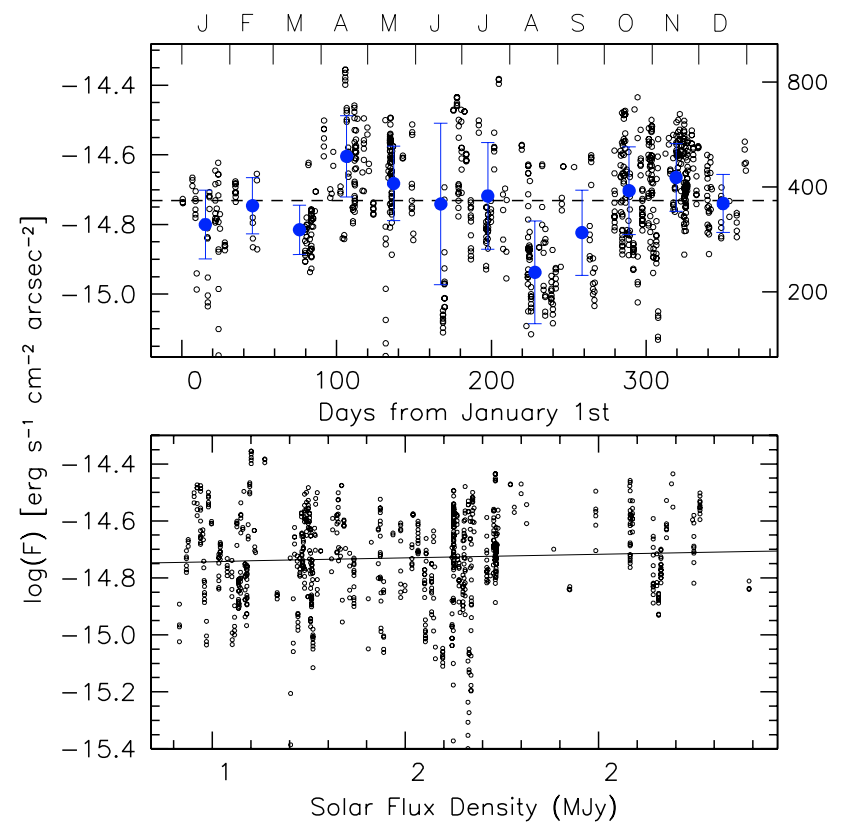

Fig. 18. Same as Fig. 9 for the $\mathrm{OH}(8-3)$ molecular band. No solar flux correction has been applied.

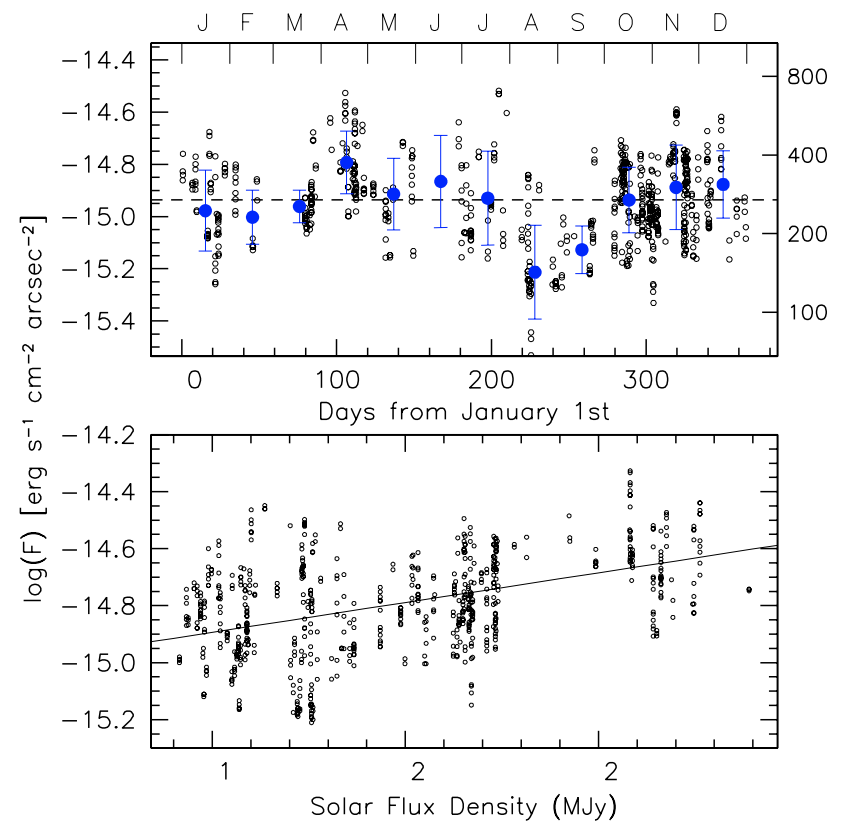

Fig. 19. Same as Fig. 9 for the $\mathrm{O}_{2}(0-1)$ molecular band.

difficult to remove. In fact, for the sake of simplicity, no differential zodiacal light contribution has been applied to the continuum measurements. This is certainly affecting the blue bands and the continuum regions, especially those close to the zodiacal light spectrum peak. This and the improper removal of airmass effects is most likely the cause for the lower correlations shown by these features and the solar activity, than what is detected, for example, for the $U$ and $B$ passbands.

The integrated flux varies by about a factor 2 around the average value, with a fairly symmetric distribution. As already indicated by the $B$ band data (see Sect. 5), there is no significant trace of an SAO for none of the blue bands (see Fig. 20, lower panel). Interestingly, while the continuum range C1 (5040-5120 A, see Table 2) also does not display any evidence of seasonal
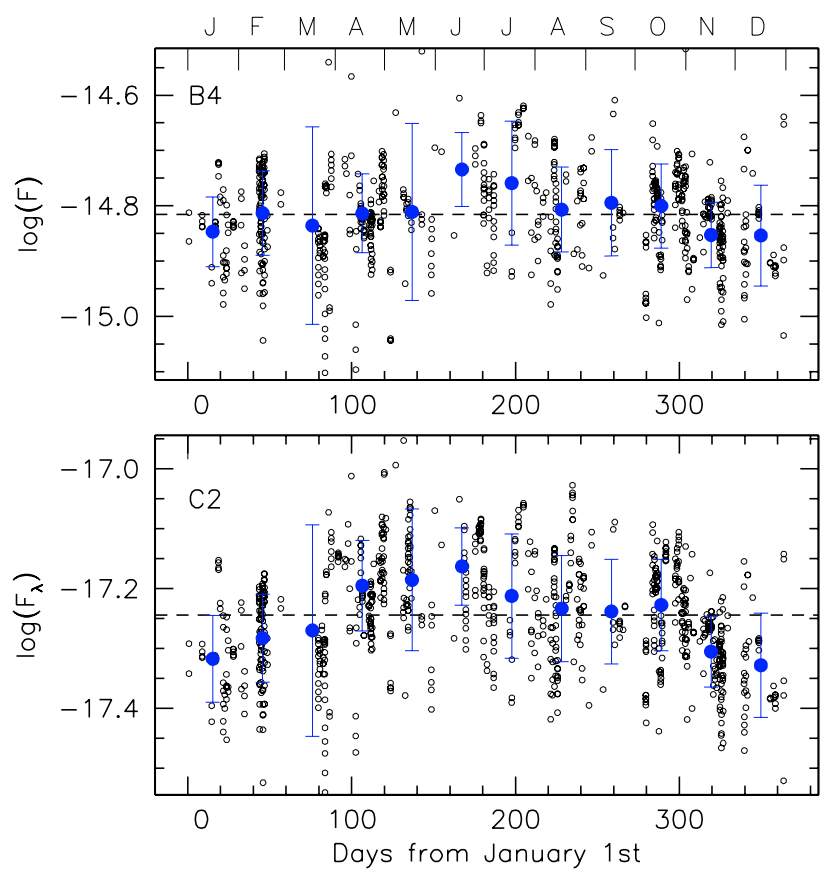

Fig. 20. Seasonal variation of blue band B4 (upper panel) and continuum range $\mathrm{C} 2$ (lower panel).

fluctuations, the redder ranges $\mathrm{C} 2$ to $\mathrm{C} 6$ show a possible broad annual oscillation, with a peak in June (Fig. 20, lower panel).

While the region between 3100 and $3900 \AA$ (mostly not covered by the data set discussed in this paper) shows a number of emission features attributable to $\mathrm{O}_{2}$ Herzberg and Chamberlain bands, the spectral interval $3900-4900$ is almost a pure pseudocontinuum (see for instance Broadfoot \& Kendall 1968, and Fig. 2 here). Nevertheless, clear variations are seen in the FORS1 database, as it is illustrated in Fig. 21, where I have compared two spectra obtained with the same instrumental setup and similar signal-to-noise ratio on two different nights. While the two spectra show the same overall emission features, these are much more pronounced in the data obtained in November 1999.

For an explorative analysis, I measured the fluxes of some of the most prominent features, which I marked in Fig. 21 (lower

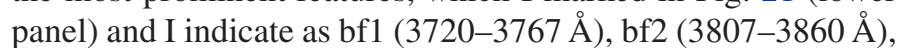
bf3 (4150-4200 ̊), bf4 (4387-4460 ̊), and bf5 (4809-4859 А). While bf1, bf 2 and bf 3 can be tentatively identified as $\mathrm{N}_{2}$ and $\mathrm{N}_{2}^{+}$molecular bands (see Chamberlain 1961, and also the next section here), the identification of bf 4 and bf5 is more difficult. For simplicity, I used a common value for the blue pseudocontinuum (CB), which was estimated in the spectral range 4730-4760 ̊. It does not show any trace of emission features (see for instance Hanuschik 2003). The results are summarised in Table 7, which reports the maximum and median values derived from the 114 suitable spectra used in the analysis, together with the percentage of non-detections.

The widest range of variation is shown by bf 1 , which can reach a maximum flux of about $1.4 \times 10^{-16} \mathrm{erg} \mathrm{s}^{-1} \mathrm{~cm}^{-2} \operatorname{arcsec}^{-2}$, while bf2 to bf5 reach peak fluxes between 6 and $8 \times$ $10^{-17} \mathrm{erg} \mathrm{s}^{-1} \mathrm{~cm}^{-2} \operatorname{arcsec}^{-2}$. Despite their similar peak values, these features display different behaviours. For instance, while bf5 is practically always detected, bf 2 is absent in more than $50 \%$ of the cases. Since the median value of the pseudocontinuum is $7.2 \times 10^{-18} \mathrm{erg} \mathrm{s}^{-1} \mathrm{~cm}^{-2} \operatorname{arcsec}^{-1}$, each of these features contributes less than $2 \%$ to the integrated flux between 3700 and $5000 \AA$. Therefore, their fluctuations can only partially 


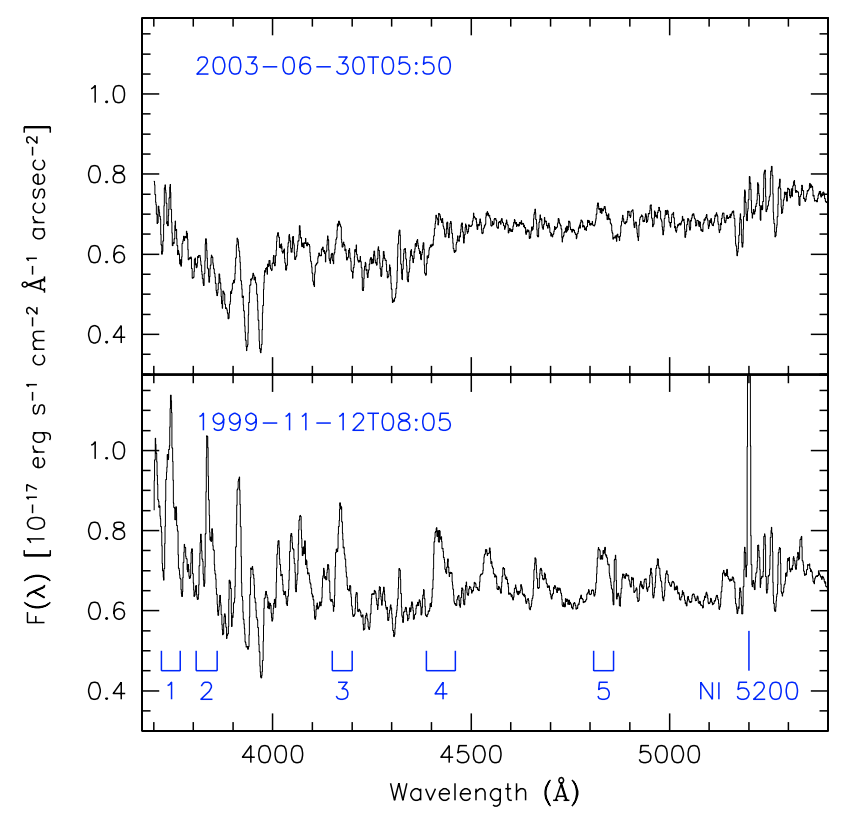

Fig. 21. The spectral region 3750-5400 $\AA$ in two different epochs. Both spectra were obtained in dark time with the $600 \mathrm{~B}$ grism. The two narrow absorptions visible between 3900 and $4000 \AA$ are the CaII H\&K solar features.

Table 7. Dark-time maximum and median fluxes for the blue emission features indicated in Fig. 21.

\begin{tabular}{cclcc}
\hline \hline $\begin{array}{c}\text { bf } \\
\#\end{array}$ & $\begin{array}{c}\text { Wavelength range } \\
(\AA)\end{array}$ & $\begin{array}{l}\max \\
\left(10^{-17} \mathrm{erg} \mathrm{s}^{-1} \mathrm{~cm}^{-2} \operatorname{arcsec}^{-2}\right.\end{array}$ & $\begin{array}{c}\text { n.d. } \\
(*)\end{array}$ \\
\hline 1 & $3720-3767$ & 14.4 & 5.4 & $26 \%$ \\
2 & $3807-3860$ & 8.4 & 0.0 & $54 \%$ \\
3 & $4150-4200$ & 6.4 & 1.3 & $38 \%$ \\
4 & $4387-4460$ & 7.2 & 2.8 & $17 \%$ \\
5 & $4809-4859$ & 8.0 & 3.9 & $1 \%$ \\
\hline
\end{tabular}

* Frequency of non-detections (flux below $10^{-17} \mathrm{erg} \mathrm{s}^{-1} \mathrm{~cm}^{-2} \operatorname{arcsec}^{-1}$ ).

account for the variations observed in the $B$ passband, which must be related to the changes in the pseudo-continuum.

\subsubsection{The strange event of 2004 November 8}

Normally, there are no conspicuous, isolated emission features in the airglow bluewards of $5200 \AA$, which is dominated by the pseudo-continuum (Roach \& Gordon 1973; see also Fig. 2 here). However, while analysing a set of low-resolution spectroscopic data obtained with FORS2 on November 8, 2004, N. Castro and M. Garcia noticed unexplained emission features between 3600 and $4400 \AA$. A more detailed analysis has shown that these emissions were present in all the FORS2 data of that night, i.e. a set of MXU exposures $2700 \mathrm{~s}$ each, obtained between 00:15 and 07:08 $\mathrm{UT}^{5}$ using the $600 \mathrm{~B}$ grism. As expected, no trace of these features was visible in a similar data set obtained three days later. An example is shown in Fig. 22 where, for comparison, an analogous spectrum obtained on November 11 is also plotted. Clearly, the two spectra differ mainly for the presence of two prominent emission bands, peaking at $3194 \AA$ and $4278 \AA$, which are identified as $\mathrm{N}_{2}^{+}$first negative bands $1 \mathrm{~N}(0-0)$ and $1 \mathrm{~N}(0-$ 1) (Chamberlain 1961; Table 5.4). These features, which are

\footnotetext{
5 On November 8, 2004 the evening twilight ended at 00:23 UT and the morning twilight started at 08:27 UT.
}

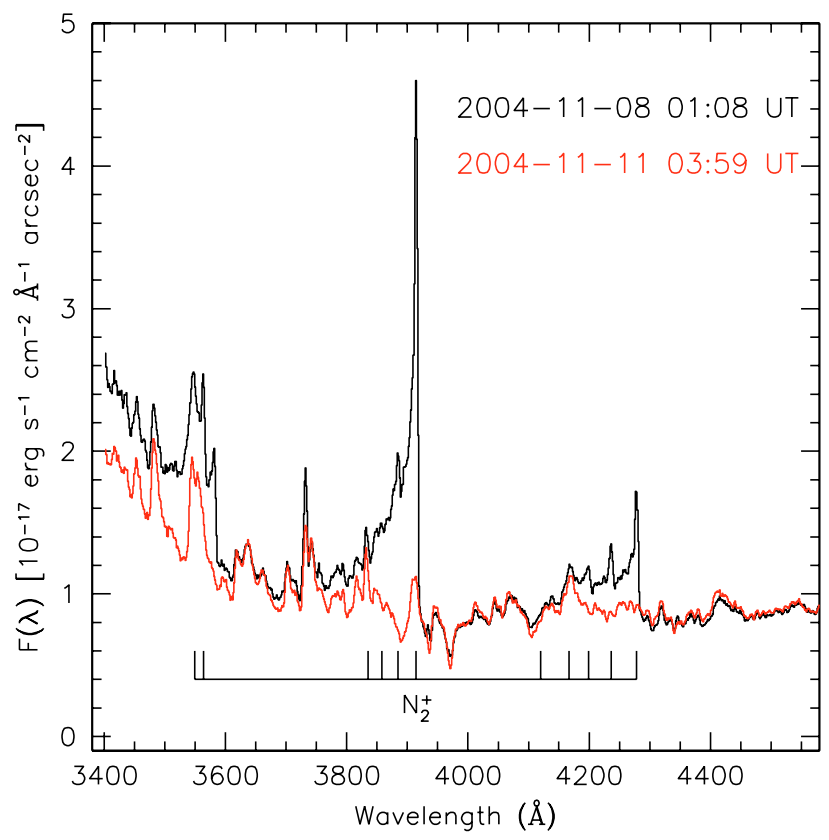

Fig. 22. The weird night sky spectrum of 2004 November 8 . The $\mathrm{N}_{2}^{+}$first negative band identifications are from Chamberlain (1961; Table 5.4). For presentation the Nov. 11 spectrum has been scaled by a factor 1.1 to match the pseudo-continuum on Nov. 8.

normally very weak or even absent in the nightglow (Broadfoot $\&$ Kendall 1968) are, on the contrary, typical of the aurora spectrum (Chamberlain 1961). Besides being an extremely strange phenomenon at the latitudes of Paranal, an aurora would certainly be accompanied by other spectral markers, such as a large increase in the emission of the [OI]5577 line, which can in fact reach an intensity of $100 \mathrm{kR}$ during an IBC III aurora (Chamberlain 1961). The flux carried by this line in the same spectrum presented in Fig. 22 is $\sim 183 \mathrm{R}$, which is slightly below the average level measured for Paranal (230 R, see Sect. 6.1.1). This definitely rules out an exceptional auroral event as being responsible for the unusual spectrum observed on November 8 , 2004.

A plausible explanation, proposed by Castro \& Garcia (private communication) is contamination by the reflection from clouds of a number of lightning strokes. In the spectral range covered by the FORS 2 data (3600-6100 $\AA$ ), the most prominent features in a lightning spectrum are the $\mathrm{N}_{2}^{+}$first negative bands $1 \mathrm{~N}(0-0)$ and $1 \mathrm{~N}(0-1)$ (see for instance Wallace 1964). Additionally, on the night of 2004 November 8 thick and thin cirrus were reported in the ESO-Paranal night logs, substantiating the hypothesis of scattered light from a fairly distant thunderstorm. This kind of events must indeed be very rare, since no other example could be found in the FORS1 spectral data base presented in this paper.

\section{Correlations between spectral features}

For a first exploratory analysis I computed the linear correlation coefficients in the $\log F-\log F$ plane between all measured features. The results are presented in Table 8 , and they basically confirm the correlations found by Barbier (1956), even though new interesting facts do appear.

The pioneering optical, eight-colour photometric studies by Barbier (1956) have shown the existence of the so-called covariance groups: the green-line group ([OI]5577, $\mathrm{O}_{2}$ Herzberg 

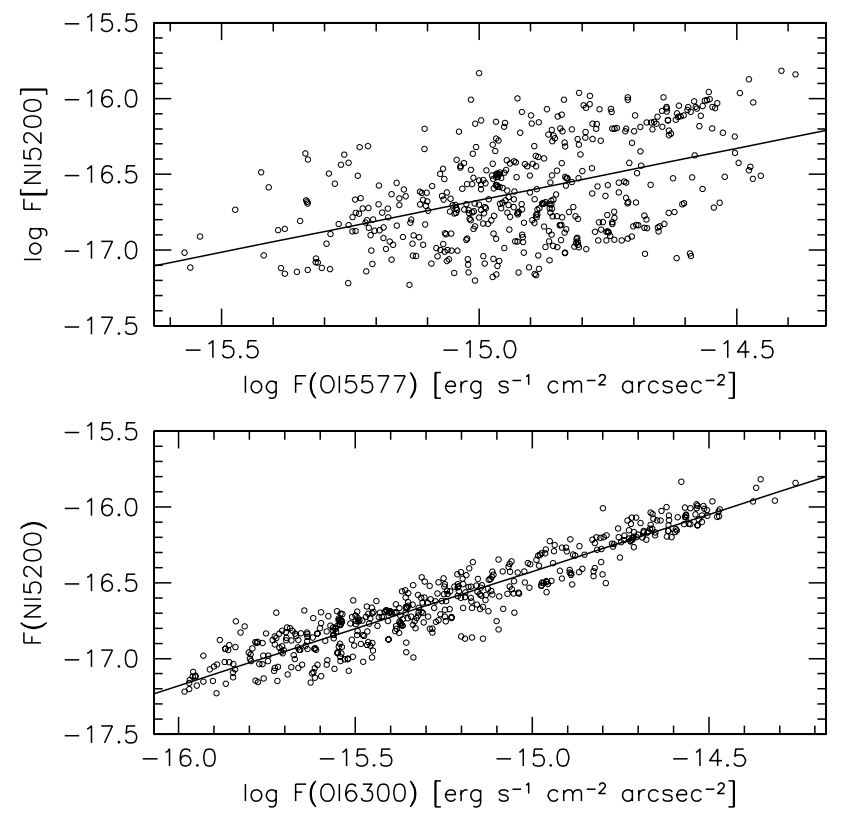

Fig. 23. Correlation between N I 5200 and [OI]5577 (upper panel) and [OI]6300 (lower panel). Only airmass correction has been applied.

bands, the blue bands, the green continuum and the $\mathrm{O}_{2}(0-1)$ band), the sodium group ( $\mathrm{Na}$ I D doublet and the $\mathrm{OH}$ bands), and the red-line group, which includes only the [OI]6300, 6364 doublet. So far, the latter has appeared to be completely independent of any other component of the airglow (see Chamberlain 1961). Nevertheless, as shown in Fig. 23, [OI]6300 shows a very tight correlation with the N I feature at $5200 \AA$. The linear correlation factor in the log-log plane is $r=0.95$ and this appears to be one of the strongest correlations between airglow features found in the data set presented here, surpassed only by what is shown by the $\mathrm{OH}$ bands (see Table 8). To my knowledge, this is the first time this finding has ever been reported; most likely, it has escaped the attention of previous investigations simply because the $\mathrm{N}$ I feature is rather weak $(\leq 30 R)$, hence practically impossible to measure with intermediate passband filters.

Even though a correlation between N I 5200 and [OI]5577 is found (see Fig. 23, upper panel), this is less marked $(r=0.56)$ and the spread around the best-fit relation is much greater $(\sigma=$ 0.29 vs. $\sigma=0.11$ ). Finally, the correlation between [OI]5577 and [OI]6300 is indeed weak ( $r=0.29, \sigma=0.39)$; nevertheless, the data presented here seem to indicate that, on average, the maximum value attained by the red line is related to the flux of the green line through the simple relation $\mathrm{F}([\mathrm{OI}] 6300) \leq 2 \times$ $\mathrm{F}([\mathrm{OI}] 5577)$.

The strongest correlation within the [OI]5577 covariance group is that with the $\mathrm{O}_{2}$ band $(r=0.85)$, followed by the blue bands B1 $(r=0.61)$, N I $5200 \AA(r=0.56)$, B2 $(r=0.55)$, C3 $(r=0.43)$ and C2 $(r=0.41)$. The correlation with the other continuum regions is weaker $(r \leq 0.4)$. In the Na I D group, besides the very tight correlations existing between the $\mathrm{OH}$ bands $(r \geq 0.97)$, the strongest correlation is observed between Na I D and $\mathrm{OH}(8-3)(r=0.75)$, followed by the other $\mathrm{OH}$ bands. Na I D correlates rather well with the continuum ranges $\mathrm{C} 2(r=0.58)$ and C6 $(r=0.63)$ and with the $\mathrm{O}_{2}$ band $(r=0.61)$. As anticipated in the previous section, the only meaningful correlation found in the third covariance group is between [OI]6300 and the $\mathrm{N}$ I 5200 line $(r=0.95)$. This is actually one of the tightest correlations found among all features. Finally, all continuum bands
C1 to C6 are well correlated with each other and with the blue bands B1 to B4, which show a very strong mutual correlation as well.

Even though the analysis is certainly hampered by the smaller sample, I have run a similar exploratory study for the blue emission features described in Sect. 6.3. The results are presented in Table 9. The first interesting fact is that the blue emission features bf 1 to bf 4 are uncorrelated with the $\mathrm{CB}$ continuum $(|r| \leq 0.15)$, while some correlation is seen for bf5 ( $r \simeq 0.4)$. On the other hand, all blue features show a significant mutual correlation, which ranges from a minimum $(r \simeq 0.4)$ for bf 2 and bf5 to a maximum ( $r \simeq 0.9$ ) for bf 3 and bf4. In general, bf5 is the feature that shows the weakest correlation with the remaining blue features. Additionally, it displays the strongest correlation with the $[\mathrm{OI}] 5577$ feature $(r \simeq 0.8)$ and solar activity. Indeed, the correlation with [OI] 5577 is rather marked for all bf's $(r \geq 0.5)$, suggesting that these features might belong to the green-line group of Barbier (1956). Nevertheless, bf 1 and bf2 show a similarly marked correlation to the N I 5200 line $(r \geq 0.5)$, which belongs to the red line group. This suggests a partial correlation between the abrupt micro-auroral events undergone by the [OI]6300, $6364 \AA$ doublet and the blue features activity.

As far as the correlation with solar activity is concerned, it is interesting to note that, while the blue pseudo-continuum shows a very similar correlation factor with $S F D_{\mathrm{d}}$ and $S F D_{\mathrm{m}}$, this is significantly larger for the blue features bf 2 and bf 3 when the daily averages are used. In contrast, bf5 shows a stronger correlation with the monthly averages. Because of the limited sample, though, these results have to be taken with some caution. In fact, for instance, the [OI]5577 line shows a suspiciously high correlation factor with $S F D_{\mathrm{d}}(0.54)$, at variance with the value derived from the whole data set $(0.30$; see Table 8$)$.

\section{Short time-scale variations}

In general, the spectroscopic time coverage of the present sample is sparse, making the study of short time-scale variations (minutes to hours) rather difficult. Interestingly, the sample presented here includes some data sets for which the spectroscopic observations went on for a significant fraction of the night. The most complete case is illustrated in Fig. 24, which presents the behaviour of some selected features during the night of 200104-22 as a function of time elapsed from the end of evening twilight, with an average interval of thirty minutes. This sequence definitely shows that the variations are smooth, with typical time-scales of the order of several hours. As expected, the most marked change is seen in the [OI]6300 feature that, during the first hours of the night, went through one of the intensity enhancements typical for sites close to the geomagnetic equator.

The smooth time evolution seen in the emission features matches the behaviour observed in broad-band photometry when long time series are available (see Paper I, Sect. 6).

\section{Discussion}

The analysis presented in this paper gives a clear picture of the complexity shown by the nightglow fluctuations, most of which remain unexplained. If it is well-established that several features show a definite correlation with solar activity, to which they react on time-scales of a couple of weeks, more thorough investigations need to be performed in order to better understand the link between space weather and the phenomena taking part in the upper layers of Earth's atmosphere. 
Table 8. Linear correlation factors in the $\log F-\log F$ plane.

\begin{tabular}{|c|c|c|c|c|c|c|c|c|c|c|c|c|c|c|c|c|c|c|c|c|c|c|}
\hline & 1 & 0 & 22 & ) & 3 & 5577 & C4 & 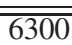 & C5 & (6- & $7-$ & (8-3) & C6 & $(6-$ & $\mathrm{O}_{2}$ & B1 & B2 & 33 & B4 & Bro & $S F D_{\mathrm{c}}$ & SFL \\
\hline C1 & & 0.15 & 0.79 & 0.14 & 0.94 & 0.31 & 0.82 & 0.02 & 0.88 & 0.23 & 0.14 & 0.15 & 0.67 & 0.14 & 0.16 & 0.87 & 0.95 & 0.99 & 1.00 & 0.60 & 0.25 & 0.23 \\
\hline 5200 & 0.15 & & 0.36 & 43 & 0.29 & 56 & 0.31 & 95 & 22 & 0.34 & 0.32 & 0.32 & 5 & 0.29 & 0.21 & 31 & 22 & 16 & 0.17 & 0 & 31 & .45 \\
\hline $\mathrm{C} 2$ & 79 & .36 & & 0.58 & 0.94 & 0.41 & 0.99 & 0.28 & 96 & 0.40 & 0.32 & 0.37 & 0.92 & 0.46 & 0. & 0.75 & 77 & 76 & 0.79 & 90 & 2 & .14 \\
\hline $\mathrm{Na}$ ID & 14 & 0.43 & 0.58 & & 0.35 & 0.41 & 0.52 & 0.32 & 0.41 & 0.70 & 0.72 & 0.75 & 0.63 & 0.61 & 0.61 & 0.19 & 0.14 & 0.15 & 0.16 & 0.72 & .03 & 0.07 \\
\hline $\mathrm{C} 3$ & 0.94 & .29 & 0.94 & 0.35 & & 0.43 & 0.97 & 0.17 & .98 & 0.21 & 0.12 & 0.16 & 0.86 & 0.33 & 0.32 & 0.85 & 0.89 & 0.92 & 0.94 & 0.80 & 0.23 & 0.22 \\
\hline 5577 & 0.31 & .56 & 0.41 & 0.41 & 0.43 & & 0.38 & 0.29 & 0.31 & 0.31 & 0.29 & 0.32 & 0.26 & 0.25 & 0.85 & 0.61 & 0.55 & 0.37 & 0.36 & 0.59 & 0.30 & 0.49 \\
\hline $\mathrm{C} 4$ & 0.82 & 31 & 0.99 & 0.52 & 0.97 & 0.38 & & 0.26 & 0.99 & 0.36 & 0.28 & 0.32 & 0.93 & 0.44 & 0.33 & - & - & 0.81 & 0.83 & 0.90 & 13 & 0.13 \\
\hline 6300 & 0.02 & 95 & 0.28 & 0.32 & 0.17 & 0.29 & 0.26 & & 0.19 & 0.15 & 0.13 & 0.13 & 0.28 & 0.15 & 0.01 & - & 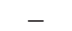 & 0.02 & 0.02 & 0.49 & 09 & .23 \\
\hline C5 & 38 & 22 & 0.96 & 0.41 & 0.98 & 31 & 0.99 & 0.19 & & 0.27 & 0.19 & 0.23 & 0.91 & 0.37 & 0.26 & - & 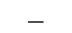 & 0.87 & 0.88 & 0.83 & 4 & .10 \\
\hline -1) & 23 & 34 & 40 & 0.70 & 0.21 & 31 & 0.36 & 0.15 & 0.27 & & 0.98 & 0.97 & 0.67 & 0.97 & 0.53 & - & - & 0.25 & 0.25 & 51 & 2 & .07 \\
\hline-2$)$ & 4 & 32 & 32 & 0.72 & 0.12 & 29 & 0.28 & 0.13 & 0.19 & 0.98 & & 1.00 & 0.63 & 0.99 & 0.57 & 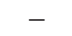 & 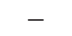 & 17 & 0.17 & 55 & 06 & 0.04 \\
\hline$(8-3)$ & 15 & 32 & 37 & 0.75 & 0.16 & 32 & 0.32 & 13 & 23 & 0.97 & 1.00 & & 0.65 & 0.98 & 0.60 & - & - & 18 & 0.18 & 9 & 04 & 0.06 \\
\hline C6 & 67 & 35 & 92 & 0.63 & 0.86 & 26 & 0.93 & 28 & 0.91 & 0.67 & 0.63 & 0.65 & & 0.64 & 0.34 & - & - & 0.68 & 0.68 & 0.9 & 02 & 0.05 \\
\hline$(6-2)$ & 14 & 29 & .46 & 0.61 & 0.33 & 25 & 0.44 & 15 & 0.37 & 0.97 & 0.99 & 0.98 & 0.64 & & 0.47 & - & - & 0.16 & 0.16 & 0.63 & .06 & 0.10 \\
\hline $\mathrm{O}_{2}$ & 6 & 21 & 0.35 & 0.61 & 0.32 & 85 & 0.33 & 0.01 & 0.26 & 0.53 & 0.57 & 0.60 & 0.34 & 0.47 & & - & - & 0.24 & 0.22 & 0.52 & 0.22 & 0.41 \\
\hline B1 & & 31 & 75 & 0.19 & 0.85 & 61 & - & - & - & - & - & - & - & - & - & & 0.96 & 0.91 & 0.89 & - & 0.28 & 0.25 \\
\hline B2 & & 22 & 77 & 0.14 & 0.89 & & - & - & - & - & - & - & - & - & - & 0.96 & & 0.99 & 0.97 & - & 0.28 & 0.24 \\
\hline B3 & $\Omega_{0}$ & 16 & 76 & 0.15 & 0.92 & 37 & 0.81 & 0.02 & 0.87 & 0.25 & 0.17 & 0.18 & 0.68 & 0.16 & 0.24 & 0.91 & 0.99 & & 0.99 & 0.61 & 0.24 & 0.25 \\
\hline B4 & 0 & 17 & 79 & 0.16 & 0.94 & 0.36 & 0.83 & 0.02 & 0.88 & 0.25 & 0.17 & 0.18 & 0.68 & 0.16 & 0.22 & 0.89 & 0.97 & 0.99 & & 0.62 & 0.27 & 0.26 \\
\hline Broad & 60 & 60 & .90 & 0.72 & 0.80 & 59 & 0.90 & 0.49 & 0.83 & 0.61 & 0.55 & 0.59 & 0.90 & 0.63 & 0.52 & - & - & 0.61 & 0.62 & & 0.14 & 0.24 \\
\hline$S F D_{\mathrm{d}}$ & 0.25 & 31 & 0.12 & 0.03 & 0.23 & 30 & 0.13 & 0.09 & 0.14 & 0.02 & 0.06 & 0.04 & 0.02 & 0.06 & 0.22 & 0.28 & 0.28 & 0.24 & 0.27 & 0.14 & & .71 \\
\hline$S F D_{\mathrm{m}}$ & 23 & 45 & 0.14 & 0.07 & 0.22 & 49 & 0.13 & 0.23 & 0.10 & 0.07 & 0.04 & 0.06 & 0.05 & 0.10 & 0.41 & 0.25 & 0.24 & 0.25 & 0.26 & 0.24 & 0.71 & \\
\hline
\end{tabular}

Note: Only dark time data have been used. The last two columns and rows report the correlation with daily $\left(S F D_{\mathrm{d}}\right)$ and monthly $\left(S F D_{\mathrm{m}}\right)$ solar flux density averages in the semi-log plane.

Table 9. Same as Table 8 for the blue features.

\begin{tabular}{l|cccccccccc|cc}
\hline \hline & C1 & 5200 & C3 & 5577 & CB & bf1 & bf2 & bf3 & bf4 & bf5 & $S F D_{\mathrm{d}}$ & $S F D_{\mathrm{m}}$ \\
\hline C1 & & 0.21 & 0.97 & 0.40 & 1.00 & 0.05 & 0.01 & 0.15 & 0.15 & 0.40 & 0.32 & 0.30 \\
5200 & 0.21 & & 0.32 & 0.59 & 0.20 & 0.56 & 0.51 & 0.21 & 0.12 & 0.13 & 0.49 & 0.46 \\
C3 & 0.97 & 0.32 & & 0.48 & 0.96 & 0.15 & 0.19 & 0.23 & 0.07 & 0.46 & 0.38 & 0.36 \\
5577 & 0.40 & 0.59 & 0.48 & & 0.38 & 0.65 & 0.47 & 0.54 & 0.56 & 0.80 & 0.54 & 0.45 \\
CB & 1.00 & 0.20 & 0.96 & 0.38 & & 0.05 & 0.00 & 0.15 & 0.14 & 0.38 & 0.30 & 0.28 \\
bf1 & 0.05 & 0.56 & 0.15 & 0.65 & 0.05 & & 0.82 & 0.76 & 0.54 & 0.50 & 0.39 & 0.30 \\
bf2 & 0.01 & 0.51 & 0.19 & 0.47 & 0.00 & 0.82 & & 0.80 & 0.69 & 0.44 & 0.32 & 0.08 \\
bf3 & 0.15 & 0.21 & 0.23 & 0.54 & 0.15 & 0.76 & 0.80 & & 0.91 & 0.62 & 0.21 & 0.02 \\
bf4 & 0.15 & 0.12 & 0.07 & 0.56 & 0.14 & 0.54 & 0.69 & 0.91 & & 0.69 & 0.26 & 0.18 \\
bf5 & 0.40 & 0.13 & 0.46 & 0.80 & 0.38 & 0.50 & 0.44 & 0.62 & 0.69 & & 0.50 & 0.32 \\
\hline$S F D_{d}$ & 0.32 & 0.49 & 0.38 & 0.54 & 0.30 & 0.39 & 0.32 & 0.21 & 0.26 & 0.50 & & 0.79 \\
$S F D_{\mathrm{m}}$ & 0.30 & 0.46 & 0.36 & 0.45 & 0.28 & 0.30 & 0.08 & 0.02 & 0.18 & 0.32 & 0.79 & \\
\hline
\end{tabular}

In all the studies of the nightglow in the astronomical context, the radio flux at $10.2 \mathrm{~cm}$ has been used as the only proxy for the solar activity. Nevertheless, other transient solar phenomena might have some impact on the night-sky brightness, like flares, coronal mass ejections and solar proton events (see Hanslmeier 2007, for a very recent review on solar phenomena). During these events, large amounts of energetic, charged particles are released and, when they interact with the Earth's magnetic field, they cause a series of geomagnetic effects, including aurorae. Therefore, charged particles are potentially responsible for at least some of the observed nightglow fluctuations, including the SAO.

Even though this will require a dedicated analysis, I have run a preliminary study using the proton flux measured by the CELIAS/MTOF Proton Monitor (Ipavich et al. 1998) onboard $\mathrm{SOHO}^{6}$, orbiting on the Earth-Sun line at about $1.5 \times 10^{6} \mathrm{~km}$ away from Earth. The proton number flux (PNF) was derived multiplying the proton density by the proton velocity measured by CELIAS/MTOF and it is plotted in Fig. 25 for the relevant time interval (upper panel).

\footnotetext{
${ }^{6}$ Data can be downloaded from http://umtof. umd.edu/pm/crn/
}

The PNF average value during this time span is $2.6 \times$ $10^{8} \mathrm{~cm}^{-2} \mathrm{~s}^{-1}$ (corresponding to a mass-loss rate of $\sim 2 \times$ $10^{-14} M_{\odot} \mathrm{yr}^{-1}$ ), while the yearly averages range from 1.9 to $3.5 \times 10^{8} \mathrm{~cm}^{-2} \mathrm{~s}^{-1}$. The maximum average value was reached in July 2002, i.e. some time after the secondary maximum seen in the radio flux. The proton velocity ranges from 270 to $1000 \mathrm{~km} \mathrm{~s}^{-1}$ (median value $440 \mathrm{~km} \mathrm{~s}^{-1}$ ), while the number density ranges from 0.1 to $73 \mathrm{~cm}^{-3}$ (median value $4.7 \mathrm{~cm}^{-3}$ ). Looking in more detail at the SOHO data, one notices that a number of isolated and short-duration peaks are present in the PNF. In fact, in some cases, values as high as $4 \times 10^{9} \mathrm{~cm}^{-2} \mathrm{~s}^{-1}$ are reached. Already a look at Fig. 25 (upper panel) shows that the frequency of these spikes is higher closer to the solar maximum. This can be seen more quantitatively in the bottom panel of Fig. 25, where I plotted the rate of what I denote as proton events $(\mathrm{PE})^{7}$. In this context, a PE was defined as a group of adjacent CELIAS/MTOF measurements with PNF $\geq 7 \times 10^{8} \mathrm{~cm}^{-2} \mathrm{~s}^{-1}$.

In principle, since the solar rotation axis is inclined by about 7.1 degrees with respect to the ecliptic (Balthasar et al. 1987)

\footnotetext{
7 Note that these events differ from the solar proton events, which are bursts of relativistic protons, with energies larger than $10 \mathrm{MeV}$.
} 


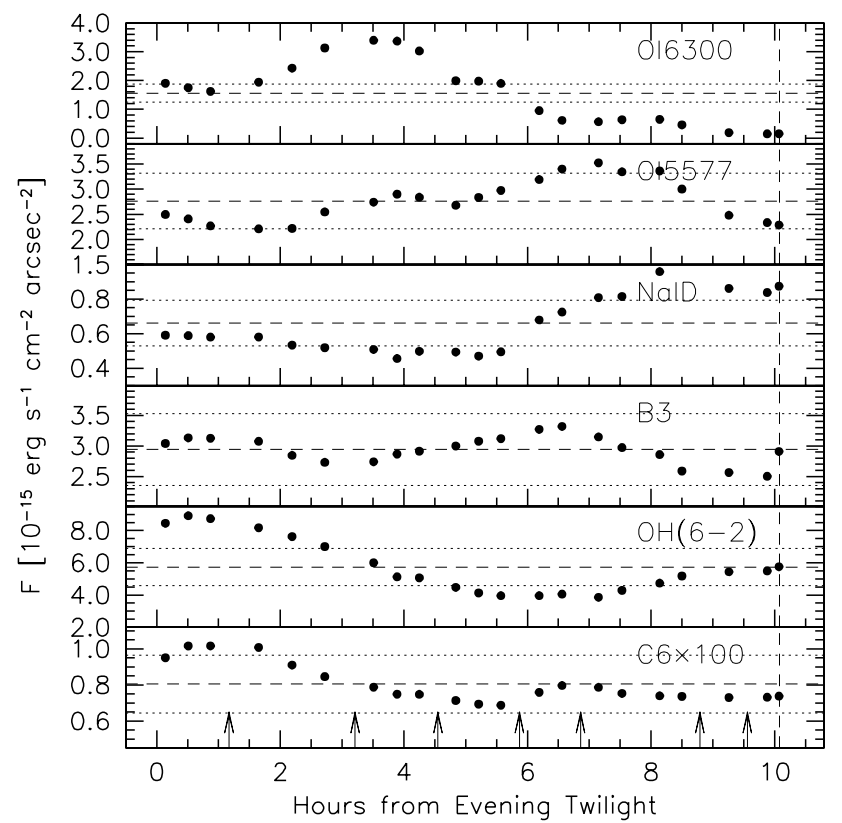

Fig. 24. Sequence of spectral measurements for some of the features studied in this work. The data were obtained with FORS1 on 200104-22 (G300V+GG435). Exposure times ranged from 10 to $20 \mathrm{~min}$. Several sky targets at different airmasses were observed. The vertical arrows indicate the times of target change. Airmasses spanned from 1.2 to 1.9 . The vertical dashed line marks the start of morning astronomical twilight. The horizontal dashed lines mark the average value, while the dotted lines indicate $\pm 20 \%$ levels.

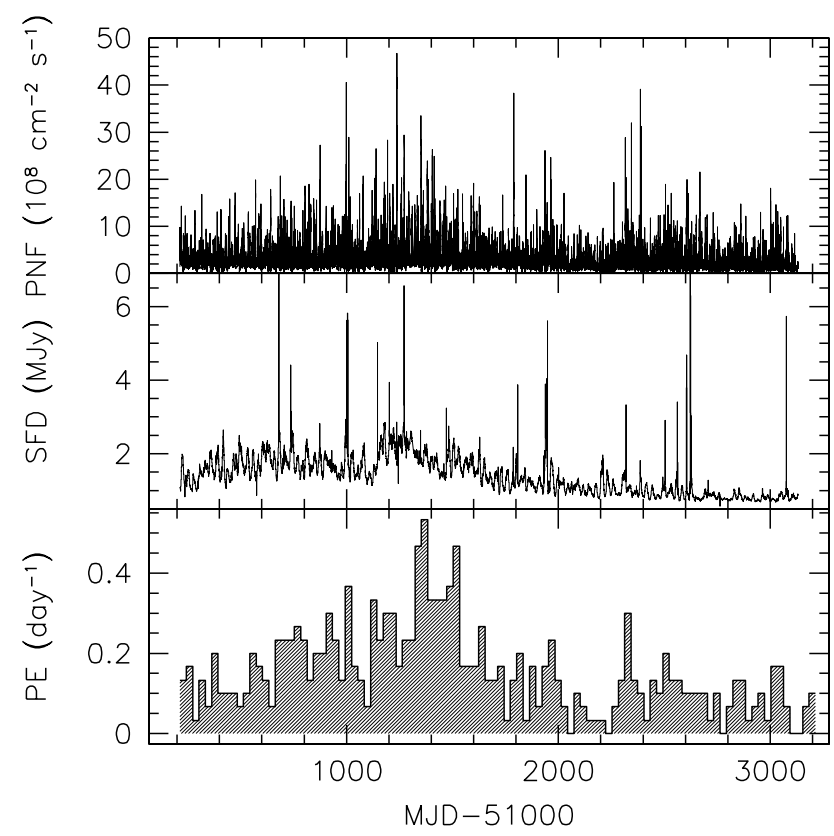

Fig. 25. Upper panel: CELIAS/MTOF proton number flux. Central panel: solar radio flux. Lower panel: CELIAS/MTOF proton events $\left(\mathrm{PF} \geq 7 \times 10^{8} \mathrm{~cm}^{-2} \mathrm{~s}^{-1}\right)$.

and because the solar wind flux is known to be higher from the poles than the equator, a modulation of the solar wind as seen from Earth is indeed expected. More precisely, the proton flux should be at its maximum around March 5 and September 5 when Earth lies at its highest/lowest heliographic latitude, respectively. For the same reason, the proton flux should be minimum around January 5 and June 5 . This prediction can be easily

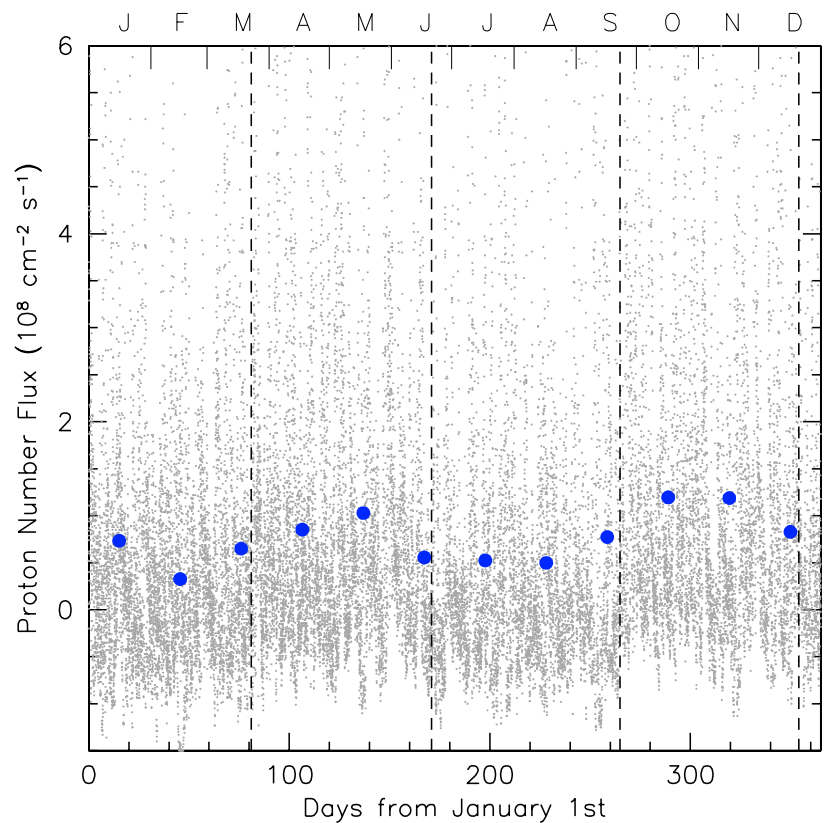

Fig. 26. Seasonal variation in the MTOF/CELIAS proton number flux. Only data from 2004-01-01 to 2007-01-01 are plotted. The filled circles trace the monthly averages. No correction for the 11-year solar cycle has been applied.

compared to the real data using the CELIAS/MTOF measurements and looking at their behaviour as a function of time elapsed since the beginning of the year. The result is shown in Fig. 26, which was produced using data obtained towards solar minimum (January 2004 to January 2007), for a total of about 28000 data points. Indeed the PNF shows a SAO, with maxima in April and November, i.e. significantly shifted in time with respect to the epochs of maximum/minimum heliographic Earth's latitude. Remarkably, the SAO observed in the PNF appears to be in phase with the SAO detected both in the broad-band data (Sect. 5) and in the emission features (Sect. 6). Interestingly, a similar plot for the $10.2 \mathrm{~cm}$ radio flux does not show any clear trace of an SAO. Also, the evidence of an SAO in the PNF for the years around the maximum of solar cycle $n .23$ becomes weaker. A possible explanation is that the more frequent and probably more stochastic PEs tend to dominate the smoother SAO when the sun is more active.

Besides the smooth, long-term variation possibly produced by the modulation of the average proton fluence, it is reasonable to think that isolated PEs might be the cause of sporadic nightglow enhancements. To explore this possibility, I ran a correlation analysis similar to the one described in Sect. 4.1 for the solar radio flux. The results are generally rather noisy and show that the correlation with PNF is always low $(|r| \leq 0.3)$, both for short $(\Delta \tau=0.5$ days $)$ and long $(\Delta \tau=30$ days $)$ time windows. An example for the $V$ passband is presented in Fig. 27 (upper panel) for an averaging window $\Delta \tau=10$ days. The correlation coefficient reaches a peak $\sim 0.3$ for $\tau \simeq 10$ days, then it decreases and suddenly drops for $\tau>27$ days. Even though the result is not really convincing, it might indicate that the response of the night-sky brightness to PEs takes place with some time delay and that what matters is the proton flux behaviour during the last solar rotation. It must be noticed that the PNF shows a rather marked recurrence, with a period of 9.1 days, which coincides with one third of the solar rotation period (see Fig. 27, lower panel). Similar to what happens for the radio data (see Sect. 4.1), 

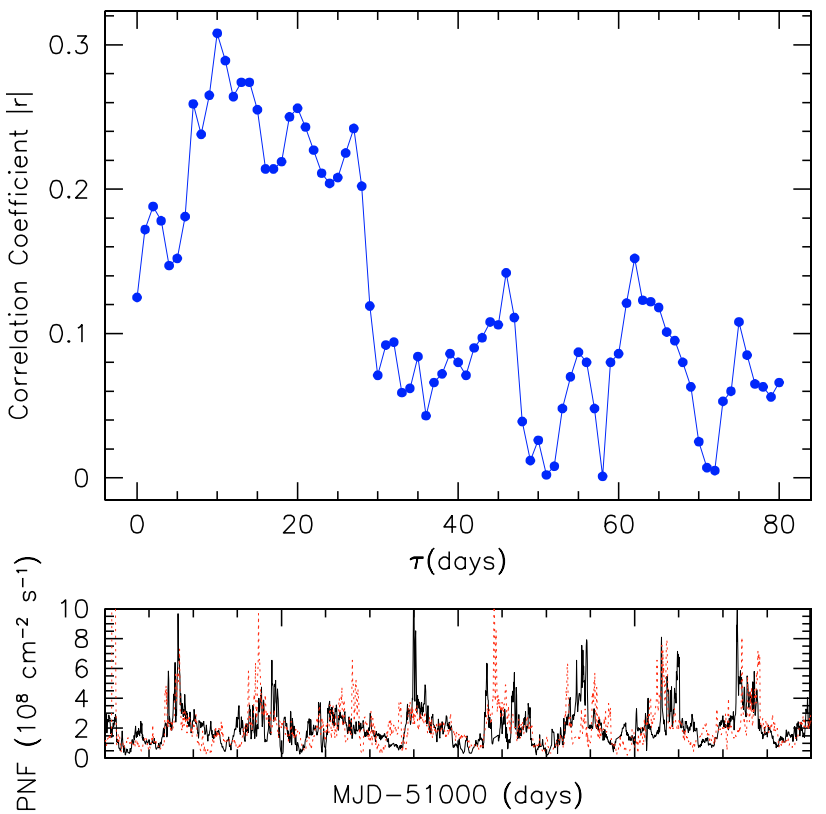

Fig. 27. Upper panel: Linear correlation coefficient between dark time $V$ sky brightness and PNF as a function of time delay $\tau$, computed for $\Delta \tau=10$ days. Lower panel: example of PNF periodicity. The dotted curve is a replica of the original data shifted by 9.1 days.

this probably creates spurious correlation peaks. Interestingly, short time-scale periodic variations in the geomagnetic activity have been detected for 13.3, 9.1, and 6.9 days (see for instance Hauska et al. 1973).

Since the typical speed of solar wind is $\sim 450 \mathrm{~km} \mathrm{~s}^{-1}$, the swarms of particles released during the PEs reach the Earth about one hour after being detected by SOHO. Therefore, the delay in the reaction is completely due to processes taking place within the Earth's atmosphere.

As a last check for intermediate time-scale periodic variations, I have investigated the correlation with the Moon motion. In fact, it has been suggested that atmospheric tides might induce recurrent oscillations in the night-sky brightness (see Chamberlain 1961 and references therein). For this purpose, I have investigated possible links between the broad-band, darktime measurements, and lunar phase or lunar hour angle. No significant correlation has been found, in agreement with a similar analysis run by Mattila et al. (1996).

\section{Conclusions}

In this paper I have presented a photometric and spectroscopic analysis of the optical night sky emission at Cerro Paranal in the time interval April 2001-January 2007. The main conclusions of this work can be summarised as follows:

- The UBVRI night-sky brightness is well-correlated with solar activity. The correlation is maximum in $U$ and minimum in $I$.

- The excursion between sunspot maximum and minimum of solar cycle n. 23 is $0.6,0.3,0.3,0.4$, and $0.2 \mathrm{mag} \mathrm{arcsec}^{-2}$ in $U, B, V, R$, and $I$ passbands, respectively.

- There are indications that the effects of solar activity on nightglow are not identical across different solar cycles.

- The reaction of the nightglow to the solar variations seems to take place with a time delay of about of 2-3 weeks.
- The $R$ night-sky brightness seems to react with a much shorter delay, of a few days.

- $V, R$, and $I$ measurements show a clear semi-annual oscillation (SAO), with a typical peak-to-peak variation of $\sim 0.5 \mathrm{mag} \operatorname{arcsec}^{-2}$. For the $B$ passband this oscillation is much smaller, if present.

- Maxima and minima of the SAO are out of phase with respect to the equinoxes and solstices.

- All main emission features show an SAO, very similar to the well-known seasonal oscillation of the Na I D doublet.

- Both [OI] 5577 and NI $5200 \AA$ show the strongest correlation with solar activity. For [OI] 5577, the maximum correlation is found for a time delay of 15 days.

- Both [OI] $6300 \AA$ and NI $5200 \AA$ show a very tight mutual correlation. Nevertheless, [OI] 6300 displays a weaker correlation with solar activity.

- Flux variations in the $\mathrm{OH}$ bands are very strongly correlated with each other and do not show any correlation with solar activity.

- The main emission features, both atomic and molecular, show smooth flux variations on time-scales of hours.

- A preliminary and exploratory analysis of the possible connection between nightglow and flux of charged particles from the Sun has shown that there is a weak correlation.

- The night-sky emission seems to react, with a delay of about 10 days, to variations in the proton number flux.

- No correlation is found between the dark-time, broad-band night-sky brightness and moon phase or moon hour angle.

Future investigations, with even larger databases, will have to address the possible relations with other solar phenomena, like coronal mass ejections, flares and solar proton events, in an attempt to connect the observed short time-scale variations of the nightglow with space weather.

Acknowledgements. I am grateful to K. Krisciunas for suggesting to investigate the time-scales of the night-sky brightness dependency on the solar activity. I also wish to thank R. Mignani, S. Möhler, and the ESO Quality Control Group for the support received during this work. Finally, I am grateful to N. Castro and M. Garcia for reporting the weird case of November 8, 2004. This paper is based on archival data obtained with ESO Telescopes at Paranal Observatory.

\section{References}

Abrams, M. C., Davis, S. P., Rao, M. L. P., Engleman, J. R., \& Brault, J. W. 1994, ApJS, 93, 351

Ageorges, N., \& Hubin, N. 2000, A\&AS, 144, 533

Balthasar, H., Stark, D., \& Wöhl, H. 1987, A\&A, 174, 359

Barbier, D. 1956, The Airglow and the Aurorae, J. Atm. Terr. Phys. Suppl., 38(5)

Barbier, D. 1957, Compt. Rend, 244, 1945

Benn, C. R., \& Ellison, S. L. 1998, La Palma Technical Note, 115

Broadfoot, A. L., \& Kendall, K. R. 1968, J. Geophys. Res., 73, 426

Buriti, R. A., Takahashi, H., \& Gobbi, D. 2001, Braz. J. Geophys., 19, 169

Chamberlain, J. W. 1961, Physics of the Aurora and Airglow (New York: Academic Press)

Covington, A. E. 1969, JRASC, 63, 125

Garstang, R. H. 1988, Observatory, 108, 159

Garstang, R. H. 1989, PASP, 101, 306

Hanuschik, R. 2003, A\&A, 407, 1157

Hanselmeier, A. 2007, The Sun and Space Weather (Springer), 2nd edition

Hauska, H., Abdel-Wahab, S., \& Dyring, E. 1973, Phys. Scr., 7, 135

Howard, R. 1999, in Allen's Astrophysical Quantities, ed. A. N. Cox (New York: AIP Press; Springer), 4th edition

Ipavich, F.M., et al. 1998, J. Geophys. Res., 103, 17205

Kirkhoff, V. 1986, Can. J. Phys., 64, 1664

Krisciunas, K. 1990, PASP, 102, 1052

Krisciunas, K. 1997, PASP, 109, 1181

Krisciunas, K., Volker, W. J. H., Semler, D. R., Richards, J., et al. 2007, PASP, 119,687

Landolt, A. U. 1992, AJ, 104, 340 
Leinert, Ch., Väisanen, P., Mattila, K., \& Lehtinen, K. 1995, A\&AS, 112, 99 Leinert, Ch., Bowyer, S., Haikala, L. K., et al. 1998, A\&AS, 127, 1

Levasseur-Regourd, A. C. \& Dumont, R. 1980, A\&A, 84, 277

Liu, Y., Zou, X., Sun, W., Ma, J., Wu, H., Jiang, Z., Xue, S., \& Chen, J. 2003, PASP, 114, 495

Lockwood, G. W., Floyd, R. D., \& Thompson, D. T. 1990, PASP, 102, 481

Mattila, K., Väisänen, P., \& Appen-Schnurr, G. F. O. 1996, A\&AS, 119, 153

Meinel, A. B., Negaard, B. J., \& Chamberlain, J. W. 1954, J. Geophys. Res., 59, 407

Patat, F. 2003a, A\&A, 400, 1183 (Paper I)

Patat, F. 2003b, A\&A, 401, 797

Pilachowski, C. A., Africano, J. L., Goodrich. B. D. \& Binkert, W. S. 1989, PASP, 101, 707
Press, W. H., Teukolski, S. A., Vetterling, W. T., \& Flannery, B. P. 1992, Numerical Recipes, 2nd edition (Cambridge: Cambridge University Press), 630

Rayleigh, L. 1928, Proc. Roy. Soc. London, Ser. A, 119, 11

Roach, F. E., \& Gordon J. L. 1973, The light of the night sky (Boston: Dordrecht Reidel)

Rosenberg, N., \& Zimmerman, S. P. 1967, Planet. Space Sci., 15, 863

Schneeberger, T. J., Worden, S. P., \& Beckers, J. M. 1979, PASP, 91, 530

Szeifert, T. 2002, FORS1+2 User's Manual, VLT-MAN-ESO-13100-1543, Issue 2.3

Slanger, T. G. et al. 2005, J. Geophys. Res., 110, D23302

Wallace, L. 1964, ApJ, 139, 994

Walker, M. F. 1988, PASP, 100, 496 